\title{
Logical models of discrete event systems: A comparative exposition
}

\author{
AMIT PATRA $^{* 1}$, SIDDHARTHA MUKHOPADHYAY ${ }^{1}$ and \\ SUPRATIK BOSE ${ }^{2}$
}

${ }^{1}$ Department of Electrical Engineering, Indian Institute of Technology, Kharagpur 721302 , India

${ }^{2}$ Centre for Artificial Intelligence and Robotics, Raj Bhavan Circle, High Grounds, Bangalore 560001 , India

e-mail: amit@ee.iitkgp.ernet.in

MS received 31 October 1995; revised 19 July 1996

\begin{abstract}
The increasing complexity of man-made systems calls for new tools and techniques to model them efficiently and at the desired level of abstraction. Well-established modelling paradigms, such as finite state machines, petri nets, communicating sequential processes etc., which are borrowed from the fields of computer science and operations research, often lack certain essential features for capturing discrete event dynamics. New tools such as state charts, timed transition models, finitely recursive processes etc., are evolving to take into account some of these requirements. In this paper we first characterize such systems as well as typical problems related to them. We then discuss and critically evaluate several modelling frameworks through examples. At the end we provide a comparison among the frameworks and directions for future research.
\end{abstract}

Keywords. Logical models; discrete event systems; continuous variable systems.

\section{Introduction and motivation}

Discrete event systems (DES) and their modelling, analysis, observation and control are receiving increasing attention in recent years. Such systems are nearly always man-made. Therefore, quite naturally, as man-made systems grow in size and complexity, need arises to evolve a systematic theory to design, operate and evaluate them. Examples of such systems include manufacturing systems, chemical processes, traffic systems, communication

* For correspondence 
networks, robotic systems etc. Such systems are characteristically viewed to possess a discrete statespace derived from associated physical variables and are asynchronous, event driven. often nondeterministic or admit choice of events by some unmodelled mechanisms. They consist of subsystems which evolve concurrently and with interactions in the form of interlocks, communications via channels or shared physical variables. The physical variables include numeric continuous variables such as a liquid level, numeric discrete variables such as machine parts in a buffer, or non-numeric variables such as traffic lights, relay flags or valve positions. Primarily the logical behaviour of the systems, namely the sequence of events that occurs, is of interest. Structural properties such as liveness, reachability etc., need to be assessed or ensured by proper design. Furthermore, often these systems are required to meet hard real-time deadlines. Such systems also arise due to state quantization in continuous variable systems (CVS).

A DES, fundamentally, is a system that has a discrete set of states in one of which it exists, and generates (or responds to) sequences of events in the course of its evolution (or operation). This easily contrasts with the CVS, that have continuous state spaces, execute continuous (at least piecewise) trajectories, and are described by ordinary or partial differential equation models. While most physical systems. either man-made or natural, behave as CVS at a sufficiently high level of resolution, it may be unnecessary to be concerned with such a description for a given purpose. For example, while designing the basic configuration of a digital circuit, one is never concerned with the analog "innards" of the integrated circuits. In fact, man-made systems are often so complex that, in order to study many aspects of their design and operation, it becomes necessary to adopt a higher level of abstraction, whereby the underlying continuous dynamics has to be completely obliterated. This is often achieved by quantizing the continuous statespace into a set of zones or discrete states, while events label the (discrete) state transitions that occur under the continuous dynamics. The need arises, therefore, for a new modelling paradigm that can represent such systems. Our efforts, in this paper, would be to introduce some of the most common modelling frameworks that have been used for this purpose.

Historically, this area has been a very active field of research during the last decade, and this trend is likely to continue in the years to come. The following arguments and facts are indicative of its inherent potential.

First, note that with increase in technological advance, the complexity of all things manmade is increasing. This emphasizes the need for theories, methods and tools to model, analyse, specify, design and verify such systems at different levels of abstraction. As more and more complex systems are built to meet new performance standards, there will be no dearth of challenging problems in this field.

Second, it is interesting to observe that, while much of control theory today is concerned with the problems of CVS, design and implementation of industrial control systems are often more concerned with problems of sequencing (e.g., programmable logic controllers), real-time distributed control systems, resource scheduling (e.g., level-3 controllers in the automation pyramid for batch processing plants, job shop type of flexible manufacturing systems) etc. Research and development in the area of DES are therefore likely to bridge this gap and should find application in a broad class of industrial control systems.

Third, the discipline of DES is assuming significantly interdisciplinary character. At present it comprises a blend of techniques mainly from the areas of computer science, 
systems and control, and operations research. Simultaneously, its application domains range from the design of micro-chips to that of computer communication systems spanning continents. Interaction among the workers from such diverse fields would definitely bring about fruitful new marriages between application and theory.

The paper is organised as follows: $\$ 2$ describes the various features of DES dynamics. The important logical models, including finite state machines, Petrinets, statecharts, timed transition models and process algebras are introduced in $\S 3$. In $\S 4$, with the help of an example drawn from the area of flexible manufacturing systems, the modelling features of the different frameworks have been illustrated. Finally $\& 5$ concludes the work with a brief comparison of the models and some directions for future work.

We refrain from calling this a survey of the field because of two reasons. First, although we have included references about all major aspects, we have not attempted an exhaustive literature review. Second, the work leaves out the large class of timed and stochastic models of DES as well as many aspects of control, observation, specification, verification etc. and concentrates just on modelling the logical behaviour of the systems.

\section{Features of the domain}

In this section we discuss the nature of the dynamics to be modelled and the nature of problems to be solved in typical contexts involving DES. Both these aspects are important for determining the appropriateness of a modelling framework for a given application as well as for understanding the motivation for different features of the framework.

\subsection{Nature of DES dynamics}

Various authors have mentioned the typical characteristics of DES dynamics (Ho 1989). We classify the features of a DES under the following heads:

(i) Asynchronism and instantaneity of events: The system trajectory is viewed as a sequence of events or state transitions. Asynchrony arises because of the fact that the components of the system that interact, including the environment, often do not possess synchronized or global clocks. There are also possibilities of random events, such as a machine breakdown, which are fundamentally asynchronous. The events are assumed to be instantaneous mainly for convenience, when one does not want to model the duration of occurrence of the event and when there is no ambiguity about the sequence of events. If necessary, one can model the duration of an event by two subevents, namely, start-event and finish-event, each of which can occur asynchronously, only maintaining the correct sequence. These features also arise naturally, when an underlying CVS exists, and a DES state is associated with, an (in)equality involving a point function of CVS signals, while DES events label transitions from one DES state to another.

(ii) Reactivity: A reactive system is one that has to respond frequently to stimuli from the environment. As a result, such systems typically possess features of real-time constraints, task preemption, prioritized interrupts etc. 
(iii) Selectivity: This implies that it has to often choose among alternatives in a deterministic or non-deterministic manner. A deterministic choice arises, when out of a set of distinct events, any one can take place, but the actual choice is made by the environment in which it is placed. On the other hand, an event taking place in a nondeterministic choice situation, is actually prompted by an internal mechanism which is not visible to the modeller.

(iv) Concurrency with communication, synchronization and interlocks: Quite often a DES contains many subsystems that evolve concurrently. However, the dynamics of these subsystems are invariably interrelated, since all are required to work for the same overall objective. The concurrent operation then appears as an interleaving of the individual event sequences. The interrelations restrict the possible interleavings among the individual sequences. Thus a particular task may require joint participation of two or more components, in which case, the corresponding events in the respective processes get synchronized. Similarly, an interlock may prevent or block the occurrence of an event in a subprocess until another event occurs in another given subprocess. Interlocks are often needed when a single resource is to be shared among a number of processes. Communication among the subprocesses is necessary to ensure synchronization or blocking. This can take place either as direct broadcast or point-to-point communication or indirectly via shared variables.

(v) Modularity and hierarchical features: These are typical means of handling large and complex systems, and man-made systems almost always exhibit these features. Modularity results when a system is decomposed into units that are close to autonomous, have well-defined input-output interfaces and perform a complete well-defined subtask. Hierarchy is an attempt to aggregate these modules, retaining sufficient details for a given problem, and only allowing the flow of the minimum necessary information from a lower to a higher level.

\subsection{Typical problems}

Here we discuss some of the typical problems that are sought to be addressed in the context of specification, design and operation of DES arising in various application areas. While there may be several other domain-specific questions, in the following we restrict ourselves only to problems of generic significance. A good modelling framework should facilitate efficient treatments of these problems:

(i) Anatysing logical behaviour: Some important problems under this category are the following.

(a) Boundedness: This property implies that the state space of the model is finite. A finite state-space ensures that problems on the model are solvable, at least in principle. However, it is possible to describe the behaviour of the same system using both bounded and unbounded models (Cieslak \& Varaiya 1990). In such a case, the use of an unbounded model deprives one of the assurance of the existence of solutions. Therefore, before attempting to construct algorithms for solution of any problem on a class of system models, one should at least know 
whether the problem is decidable for that class. Detection of boundedness is a sufficient condition for decidability of these probiems.

(b) Reachability: One is often interested in determining whether a particular state is reachable from another or any arbitrary initial state. The state information may be coded in terms of numerical parameters. A major problem of reachability analysis is that of state explosion which leads to high computational complexities. To improve the situation, techniques have been suggested to search most probable paths (Maxemchuck \& Sabnani 1987), to exploit structure and symmetry in the model (Brave \& Heymann 1993) etc.

(c) Liveness/deadlock: A system is said to be live, if under any circumstances, there is at least one event that can take place. Deadlock is the reverse situation, where each system has halted and cannot proceed further. Deadiock arises when all component subprocesses of the system have enabled events, but they are all blocked by at least one of the other subprocesses. Such situations are obviously undesirable. Deadlock problem can be considered as a special case of reachability problem. The problem of designing computationally efficient algorithms to identify and prevent deadlocked situations has received wide attention in the process algebra and parallel programming language research community. The majority of early work on deadlock and liveness was cast in terms of resource allocation problems, as in operating systems (Holt 1972). At present, most of the work on deadlock is formulated using Hoare's CSP formalism (Chandy \& Mishra 1979; Apt et al 1980; Hoare 1985: Brookes \& Roscoe 1991) etc. A comprehensive treatment on deadlock can be found in Dathi (1989).

(ii) Program verification and real-time properties: Process algebra-based DES models (CCS, CSP, DFRP etc.) as well as the TTM model contain many general purpose programming language features and can be used as rudimentary specification languages in order to make unambiguous specifications. Elaborate specification languages like Ada, OCCAM, LUSTRE, LOTOS, ESTEREL, SIGNAL, CSML etc., have been developed, using process algebra models as the basis, and additionally providing syntax and semantics. Different program verification techniques (Floyd 1967; Hoare 1969; Manna \& Pnueli 1982) can be used to verify properties of the process algebra models. In these methods, one makes assertions about program execution, before and after every instruction, which are also known as pre-conditions and post-conditions of the instruction respectively. Inference rules are used to reason about the whole program by combining assertions about the instructions. Since, in many cases, real-time and safety-critical reactive systems are modelled using the process algebra and TTM models, real-time properties like safety, realtime constraints etc., are also verified using the program verification methods (Manna \& Pnueli 1982; Ostroff 1990).

(iii) Supervision/control: The kind of control one can exercise in a DES is mainly of a restrictive nature, since there is no mechanism to force an event to take place in general. This has been adopted to recognise the existence of events which are uncontrollable (such as faults) and therefore cannot be prevented. In absence of such events however, one can force an event to occur by preventing all other enabled events from occurring. One, therefore, is interested in knowing whether prevention of inadmissi- 
ble or non-optimal behaviour of the system is possible by systematically disabling a set of controllable events (Ramadge \& Wonham 1987a; Wonham \& Ramadge 1987, 1988; Passino 1989; Holloway \& Krogh 1990; Chung et al 1992; Kumar et al 1993b). The DES, whose task is to keep track of the dynamic evolution of the controlled DES, and decide on the control action to be exercised, is known as a supervisor. The design of a supervisor is difficult because of many reasons like nondeterminism in the plant, existence of uncontrollable events such as machine breakdown, hard time-constraints, insufficient information feedback from the plant etc. The desired behavioural pattern can be stated in several ways, for example in the form of a set of reference trajectories or by specifying a set of states to be avoided/visited infinitely often. The last requirement is sometimes related to a notion of stability of the system, and the system is said to be stabilizable if this can be achieved under control (Brave \& Heymann 1990; Özveren et al 1991; Özveren \& Willsky 1991; Kumar et al 1993).

(iv) Observation: The events taking place in a DES may not always be observed by other agents in its environment, for example, when the system is physically distributed over a vast geographical area such as communication networks. It may then be necessary to design a supervisor under partial observation. One is therefore interested in determining whether the DES is observable, i.e., whether one can determine its state from the observed sequence of events alone. If it is not possible to ensure this at every instant, it may sometimes suffice to know the state intermittently with only a finite number of events in between (Cieslak et al 1988; Lin \& Wonham 1988a; Özveren \& Willsky 1990; Bose et al 1994).

(v) Performance analysis: Apart from the problems discussed above, which are predominantly of a qualitative nature, one often needs to answer quantitative questions too. For example, in a flexible manufacturing system (FMS), one may like to know the production rate or the capacity utilization. Such quantitative measures are necessary for solving high-level problems such as production planning and resource scheduling in FMS, message routing in communication networks, traffic management in transportation systems, etc. Most often these questions are answered through extensive simulation. However, since our objective in this paper is to focus on only the logical aspects of a DES, we shall not delve into this area.

\section{Logical models of DES}

The logical models of DES can be classified broadly as state-based models, where the dynamics is determined by process states, and trace-based models, where dynamics is described in terms of the event sequences generated in the system. The state-based models considered in the following are the Finite State Machine, the Statechart, the Petrinet and the Timed Transition Model. The trace-based models are represented by the Finitely Recursive Processes. 


\subsection{Finite state machine (FSM)}

The finite state machine is perhaps the oldest and the simplest model of DES and originates from the theory of automata and languages (Hopcroft \& Ullman 1979). An FSM is formally defined to be a 5-tuple

$$
\mathcal{G}=\left(Q, \Sigma, \delta, q_{0}, Q_{m}\right),
$$

where $Q$ is the set of states, $\Sigma$ is the alphabet of events, $\delta: Q \rightarrow Q$ is the deterministic transition function, $q_{0}$ is the initial state and $Q_{m} \subseteq Q$ is a subset of states, to be called marked states. These states usually signify the completion of a task, which is not necessarily termination in the sense of "final' states. $Q$ and $\Sigma$ are always assumed to be finite. In general $\delta$ is only a partial function and $\mathcal{G}$ is equivalent to a directed graph with node set $Q$ and edges $q \rightarrow q^{\prime}\left(q, q^{\prime} \in Q\right)$ labelled $\sigma \in \Sigma$ for each triple $\left(\sigma, q, q^{\prime}\right)$ such that $q^{\prime}=\delta(q, \sigma)$. The transition function $\delta$ can be naturally extended to be defined over strings of events

$$
\delta: \Sigma^{*} \times Q \rightarrow Q
$$

where $\Sigma^{*}$ denotes the set of all finite length strings $s$ of elements of $\Sigma$, including the empty string \langle\rangle . A language over $\Sigma$ is a subset of $\Sigma^{*}$. The language generated by $\mathcal{G}$ is given by

$$
L(\mathcal{G}):=\left\{w: w \in \Sigma^{*} \wedge \exists q^{\prime}=\delta\left(w, q_{0}\right)\right\},
$$

and is necessarily prefix closed. That is, if $s \in L(\mathcal{G})$, then all prefixes of $s$ also belong to $L(\mathcal{G})$. We denote the prefix closure of a language $L$ as $\ddot{L}$. Figure 1 presents an example FSM and the language it generates is given by $\overline{\alpha(\beta+\gamma) \delta^{*}}$, where $\delta^{*}$ denotes zero or more occurrences of $\delta$. Among the elements of $\Sigma$ there exists a subset which can be prevented from occurring (disabled) by external control. By selectively disabling such events by means of a control mechanism one obtains controlled behaviour. Formally let $\Sigma_{c} \subseteq \Sigma$ be the set of controllable events and $\Gamma=\{0,1\}^{\Sigma_{c}}$ be the set of all binary assignments to the elements of $\Sigma_{c}$. Then, corresponding to each such assignment $\gamma \in \Gamma$, one obtains a 'control function' $\gamma: \Sigma_{c} \rightarrow\{0,1\}$. In particular an event $\sigma$ is said to be enabled if $\gamma(\sigma)=1$. For an uncontrolled event $\sigma \in \Sigma-\Sigma_{c}$ one can define $\gamma(\sigma)=1$ and extend the map $\gamma$ accordingly. Then for the controlled system one can define an augmented transition function

$$
\delta_{c}: \Gamma \times \Sigma \times Q \rightarrow Q
$$

such that $\delta_{c}(\gamma, \sigma, q)=(\delta(\sigma, q)$ if $\delta(\sigma, q)$ is defined and $\gamma(\sigma)=1)$ and undefined otherwise. Thus in a controlled DES the possible transitions from a given state are the

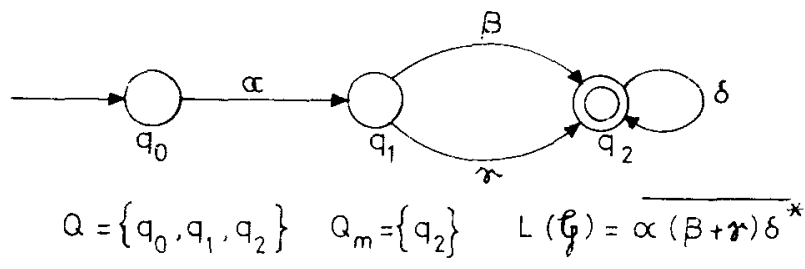

Figure 1. Example of a finite state machine. 
controllable ones which are not disabled and the uncontrollable ones, which cannot be disabled. Such a controlled DES is given by the FSM

$$
\mathcal{G}_{c}=\left(Q, \Gamma \times \Sigma, \delta_{c}, q_{0}, Q_{m}\right) .
$$

The mechanism which switches the control functions of a controlled DES to satisfy certain constraints, namely the supervisor, consists of a pair of entities given by

$$
\mathcal{S}=(S, \Phi),
$$

where

$$
S=\left(X, \Sigma, \xi, x_{0}, X_{m}\right)
$$

is a deterministic automaton, just as $\mathcal{G}$ is, with the state set $X$, input alphabet $\Sigma$, transition function $\xi: \Sigma \times X \rightarrow X$, initial state $x_{0}$ and marked subset $X_{m} \subseteq X$ and

$$
\Phi: X \rightarrow \Gamma
$$

is a total function that maps each supervisor state $x$ into a 'control pattern' $\gamma \in \Gamma$. A control pattern is a tuple of 0 and 1 , representing the control actions chosen for each controllable event. Formally for each $x \in X, \gamma:=\Phi(x) \in\{0,1\}^{\Sigma_{c}}$. $\Phi$ is known as the state feedback map.

The uncontrolled plant and the supervisor are connected in a feedback fashion (see figure 2), and each event taking place in the plant is also thought to be taking place in the supervisor synchronously. In response to each event both plant and supervisor states change, a new control pattern is chosen by the supervisor state and is applied to the plant. Often a proper supervisor is of interest where transitions are defined for every possible event in the controlled plant and every state trajectory of the closed loop process can be extended to reach the set of marked states of the plant as well as that of the supervisor.

The closed loop system involving the plant $\mathcal{G}$ and the supervisor $\mathcal{S}$ is usually denoted as $\mathcal{S} / \mathcal{G}$. The language generated by $\mathcal{S} / \mathcal{G}, L(\mathcal{S} / \mathcal{G})$ should be such that it satisfies certain objectives. However, it is not possible to achieve any arbitrarily specified language by control. Given $\Sigma$ and $\Sigma_{c}$ one can generate strings from certain controllable sublanguages of $L(\mathcal{G})$ only. A language $K \subseteq \Sigma^{*}$ is said to be controllable (with respect to $\mathcal{G}_{c}$ ) if $\bar{K} \Sigma_{u} \cap L(\mathcal{G}) \subseteq \bar{K}$ (Ramadge \& Wonham 1987a; Wonham \& Ramadge 1987), where $\bar{K}$ denotes the prefix closure of $K$. This implies that only those sublanguages of $L(\mathcal{G})$ can be generated, where strings of the form $t^{\hat{}} \sigma$ are in $\bar{K}$ with $t \in \bar{K}, \sigma \in \Sigma_{u}=\Sigma-\Sigma_{c}$. That is, a controllable language is one, for which any prefix of any string from that language, followed by an uncontrollable event, still remains in the prefix closure of the specified target (i.e., admissible) language and hence can be extended to complete strings in that

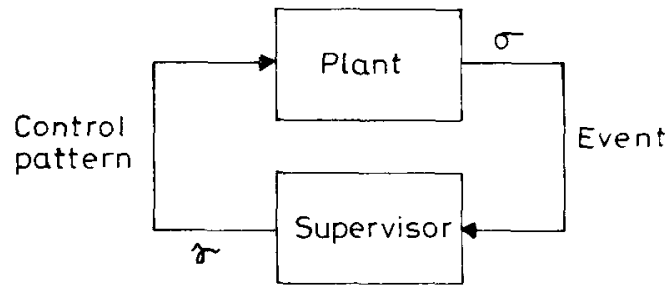

Figure 2. Closed loop configuration of a DES plant and supervisor. 
language. The supremal controllable sublanguage of $\mathrm{L}$ is defined to be the largest such language (Wonham \& Ramadge 1987)

$$
\sup C(L):=\bigcup\{K: K \subseteq L \text { and } K \text { is controllable }\} .
$$

Thus all target specification languages to be achieved by control must be subsets of sup $C(L)$. Although supervisors designed on the basis of language controllability are nonblocking, sometimes the resultant language may be too conservative and impractical, especially when the number of uncontrollable events is large. To handle such situations, the concept of an infimal controllable superlanguage has been defined and applied to the problem of supervisory control with blocking (Lafortune \& Chen 1990). Another characterisation of the language has been in terms of an infimal prefix closed and observable superlanguage (Rudie \& Wonham 1990). Further studies on such language aspects have been reported (Brandt et al 1990; Chen \& Lafortune 1991; Kumar et al 1991; Yang et al 1995).

A major disadvantage of the FSM model is that it is structurally 'flat' and the size of the state space increases exponentially with the complexity of the problem. For example when two processes operate concurrently, with only a few synchronized events, the combined system is given by a shuffle operator on the component automata and the size of the state space is of the order of the product of the sizes of that of the components. To tackle this problem a modular approach to the task of supervisor construction has been proposed (Ramadge \& Wonham 1987b; Wonham \& Ramadge 1988). This is achieved by splitting the control task into several subtasks which can be solved with the existing theory, and combining the resultant subcontrollers to obtain the solution of the original problem.

Modular supervisors can be implemented in a decentralized or hierarchical fashion. However each subsystem may require information about events in other subsystems, which may not always be accessible. This brings the question of supervision under partial observation, or that of observability. To model this situation, the observation alphabet $\Sigma_{0}$ and the projection (mask) function $P: \Sigma \rightarrow \Sigma_{0} \cup\{(\rangle\}$ are brought in. A special case is the one of natural projection, where, the unobservable events from $\left(\Sigma-\Sigma_{0}\right)$ project to the null event and therefore are simply erased from the generated string to form the observed string. Loosely speaking, a language $K \subseteq L(\mathcal{G})$ is defined to be observable, if the projection $P$ retains sufficient information to decide whether or not, after the occurrence of some observable event, the resulting string is in $K$ (Lin \& Wonham 1988a; Cieslak et al 1988).

Another characterization of observability has been given in terms of the state. A plant is said to be (state) observable if by observing a sufficiently long string in $P(L(\mathcal{G})$ ), after a finite number of events, the state of the system can be determined exactly (Özveren \& Willsky 1990; Bose et al 1994). Concepts of stability and stabilization have also been introduced in terms of state space (Brave \& Heymann 1990; Özveren et al 1991; Özveren $\&$ Willsky 1991). Although there exist minor differences in definitions of stability, most authors define a system to be stable if its trajectories go through a specified subset of the statespace infinitely often or, after entering it, remain there. More recently, however, a notion of stability has been defined in terms of the language theory (Kumar et al 1993). According to this, a language $K$ is said to be $L-$ stable, if there exists a finite integer $N^{*}$ such that $\forall s \in K$ there exists $N \leq N^{*}$ such that $s=s_{1} s_{2},\left|s_{1}\right|=N$ and $s_{2} \in L$. This implies that after a finite number of events (from the initial state), system behaviour 


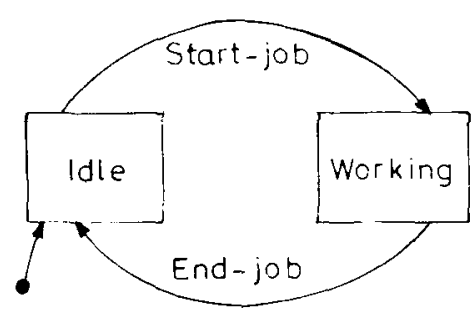

Figure 3. Finite state machine model of a robot.

conforms to the desired behaviour. This concept is similar to that of asymptotic stability of CVS.

Due to the finite state nature, all problems in this framework are decidable. Considerable work has been done to extend concepts of conventional control to this domain in the synthesis of supervisors with full state feedback (Ramadge \& Wonham 1987a, 1989; Wonham \& Ramadge 1987; Ushio 1990; Willner \& Heymann 1991) and also under partial observation (Heymann \& Lin 1993; Kozák \& Wonham 1995; Takai et al 1995). Also work has been reported dealing with decentralized supervision (Lin \& Wonham 1988b, 1990, 1991; Zhong \& Wonham 1990; Özveren \& Willsky 1992c; Rudie \& Wonham 1992), invertibility of DES (Özveren \& Willsky 1992a), tracking and restrictability (Özveren \& Willsky 1992b), diagnosability (Sampath et al 1995) etc. Work on the realisation of an FSM from a given input-output description has been reported by Kumar et al (1995). However computational complexity of algorithms is an important issue and is receiving increasing attention in recent years (Tsitsiklis 1989; Lin 1991; Rudie \& Willems 1995).

\subsection{Statecharts}

This is one of the recent approaches to specification or high level modelling of large reactive systems. It was initially developed to facilitate exact and unambiguous description of system behaviour by a large group of engineers of varying backgrounds working in a project (Harel et al 1987). Naturally the aspects that have been emphasized in this framework are ease of modelling and a co-existing hierarchy of abstraction so that models can be easily built, possibly by drawing pictures, and details of structure and functions of the various components need be considered only to the extent necessary. Quite naturally, the formalism is graphical with inherent support to multilevel hierarchical modelling. With this background, we proceed to describe its basic features.

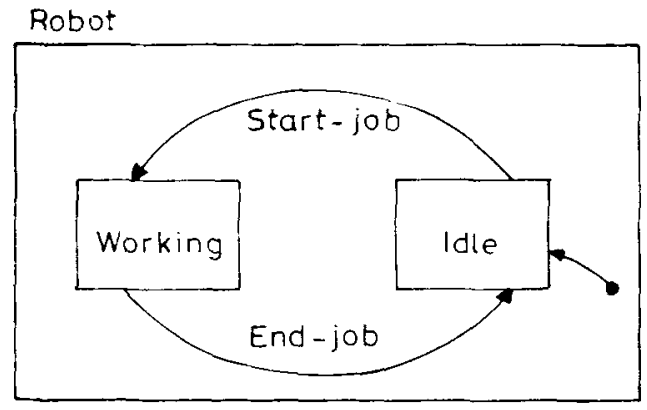

Figure 4. Statechart model of a robot. 


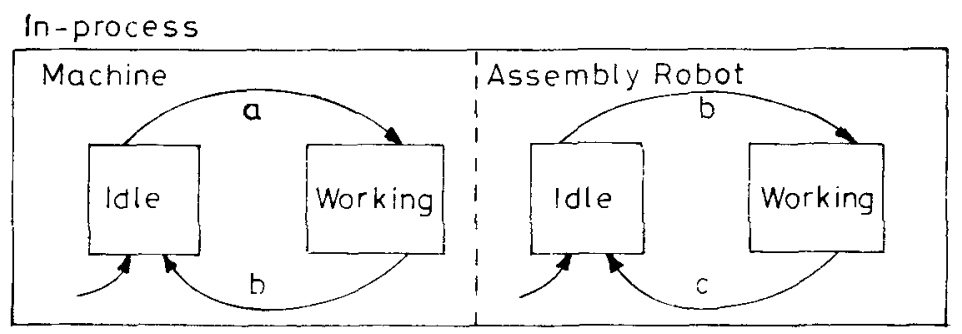

Figure 5. Statechart model of a robot-machine system.

At the lowest level of abstraction, statecharts are nothing but finite state machines. Therefore consider the simple statechart model of a machine shown in figure 3 . The external arrow into the idle state indicates that it is the initial state. The most important feature of the statechart is its support to hierarchy. To see how this is captured, consider a robot whose model is shown in figure 4 . Now let us consider the machine-robot system. This is captured by a superstate, of which the machine and the assembly-robot are orthogonal components. This means that these two components execute their dynamics concurrently. Such orthogonal subsystems are called 'AND' states. This is shown in figure 5 . Note the synchronization in the event $b$ that has been introduced to impose a certain restriction on independent concurrent behaviours of the FSMs 'machine' and 'robot'. Thus while the machine can start working anytime, the robot can do so only after the machine has finished its job and is prepared to go back to its idle state. Also, due to the synchronous nature of the event $b$, both things happen simultaneously. The overall superstate consisting of the machine and the robot may be seen at a different level of hierarchy, as one of states in which a manufacturing cell can exist. This is shown in figure 6 where the above superstate is named 'In-the arrows 'Start-process' and 'End-process' that terminate on and originate from the state 'In-process' respectively. These may be assumed to be commands to the robot and the machine. Thus the 'Startprocess' command 'resets' the whole status of the state 'In-process' such that both its components begin from their initial default states. On the other hand, the 'End-process'

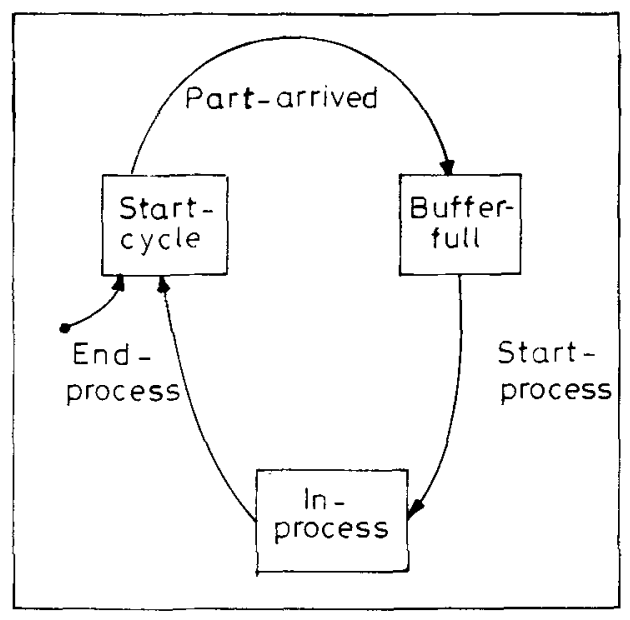

Figure 6. Statechart model of a manufacturing cell. 


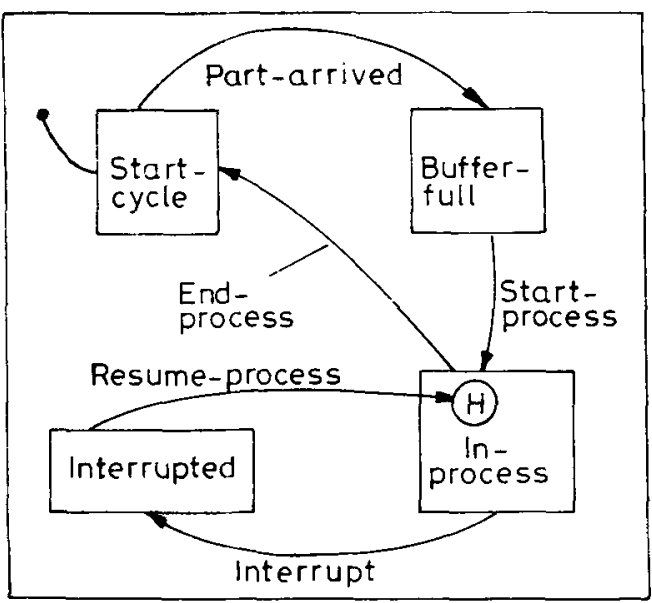

Figure 7. Statechart example of 'enter by history'.

command causes exit from the whole superstate irrespective of which individual states the machine and the robots were in. Thus the 'End-process' command stands for both normal and abnormal but forced termination. There are various types of entrance to a given state other than by default. One of them is by history, the other by condition. Entry by history is the one when the destination configuration of a transition into a superstate is determined by the component state of the superstate the system was in, when it last exited from that superstate. On the other hand, entry by condition implies the evaluation of a condition to determine the starting state. Entry by history is shown in figure 7. Further, state transitions may involve 'guards' or boolean conditions usually involving states of other concurrent components, data variables as well as logical connectivese to construct compound conditions for that enabling a transition. This is illustrated later in $\S 4$.

One of the important features of statecharts is the absence of scope restrictions on variables and events. Hence any state or data variable can be used anywhere and at any level in this formalism. Similarly, any event occurring in any component is instantly available or broadcast to all other components. While these features certainly ease modelling, the realisation of such features may pose considerable difficulties.

Statecharts offer limited support to modelling time-related features. There is a 'timeout' event of the form on-time-out $(E, T)$ which defines a new event which occurs after a duration whose value equals that of the integer expression $T$ after the occurrence of the event $E$. A second construct is that of scheduled activity denoted by schedule $(G, T)$ which schedules $G$, after $T$ time instants from the current instant. In addition, both synchronous and asynchronous simulation models are possible for statecharts.

The formalism of statecharts has not yet been standardised and many other features exist and are currently being examined for implementation. Such an implementation, called STATEMATE has been reported (Harel et al 1990; iLOGIX corporation). The formal semantics of statecharts are given by Harel et al (1987). Application of statecharts has been reported in several fields such as hardware description and synthesis (Drusinsky \& Harel 1989), aspects of hardware implementation (Drusinsky 1991) and application of the software STATEMATE to real problems (Harel et al 1990). A special form of the 
statecharts formalism has been recently introduced for tackling control and observation problems (Brave \& Heymann 1993).

In summary, the statecharts formalism is easy to use and supports many user-friendly features for modelling.

\subsection{Timed transition models (TTM)}

This is also a state-based formalism having some similarity with FSMs. Two important distinguishing features of the framework are admission of activity and data variables, and the introduction of lower and upper time bounds on state transitions. These extensions of a state machine have been made to be able to use Real-time Temporal Logic (RTTL) (Manna \& Pnueli 1982; Thistle \& Wonham 1986) to specify real-time behaviour that can be automatically verified (Clarke et al 1986). Therefore, while some compression of the state space is possible over ordinary FSMs, still the formalism inherently suffers from weaknesses of FSMs, such as lack of support for hierarchy and very large state space, as well as enjoys its strengths such as decidability of many properties and existence of verification algorithms for them. However a TTM can have infinite states mainly due to data variables.

A TTM is basically composed of three entities, namely, a set of variables which define the state of a process, a set of state transitions or events and an initial condition. An event, in turn, is parameterized by four quantities. They are :

- a boolean enabling condition function which is evaluated at every state and if the condition holds true, the associated transition is enabled.

- a state transition function that defines the transition corresponding to the event

- a lower time-bound which prevents the event from taking place before this limit is reached.

- an upper time-bound, which when reached, forces the transition to occur.

In the original TTM formalism (Ostroff \& Wonham 1990), the state transition map ( $Q \rightarrow 2^{Q}, Q$ being the state space), is allowed to be nondeterministic. Though its domain is left unspecified, it effectively gets determined by the enabling condition. To measure time there is a special event called tick. Only this event increments the time data variable $t$, usually an integer. The time bounds of all other events are specified in terms of $t$. In a departure from standard FSMs, non-unique initial states are admitted through the boolean valued initial condition. Thus any state $q$ is a valid initial state, provided $\Theta(q)$ is true, where $\Theta$ is the initial condition expression. Concurrency is supported in the formalism.

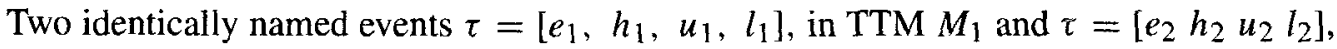
in TTM $M_{2}$, where $e_{i}$ stands for the enabling condition, $h_{i}$ stands for the state transition function, and $u_{i}$ and $l_{i}$ stand for the upper and lower time bounds respectively, may be composed as a shared event $\tau$ in synchronous operation of $M_{1}$ and $M_{2}$ as

$$
\tau=\left[e_{1} \wedge e_{2}, h_{1} \circ h_{2}, \min \left(u_{1}, u_{2}\right), \max \left(l_{1}, l_{2}\right)\right] .
$$

Nondeterminism may arise if the two functions $h_{1}$ and $h_{2}$ are non-commutative. This issue however, has not been dealt with. Additionally, explicit interprocess communication has 
been supported in the syntax through synchronized send and receive events along specific channels, much in the same way as proposed in CSP (Hoare 1985). To give an example, the two events $c ! x$ (send) and $c ? x$ (receive) occurring synchronously in two different processes indicate a communication between them involving the current value of $x$ through channel c.

Communication can also be used for sending and receiving commands. This feature may be used to model the forcing of a transition by a supervisor process. Note that at any state of the process, a number of events may be enabled, i.e., their enabling conditions may evaluate to true. Thereupon an internal choice mechanism, left unmodelled, determines the occurrence of a specified event. Events are still asynchronous and in the overall event trajectory they may be interleaved in arbitrary fashion, obeying the timing constraints, with 'tick' sequences.

To summarize, the main distinctive features of the TTM are the variables as well as the upper and lower time bounds. The time bounds may be used to answer questions related to worst/best case timings. Control of TTMs is achieved by synchronization, sharing of events or by command communications. Specification of timed behaviour may be given using RTTL (Ostroff \& Wonham 1990). Decidability of temporal logic specifications for DES has been proved (Knight \& Passino 1990; Ostroff 1990). Recently, a modified form of this formalism has been used to verify correct operation of real-time control systems (Lawford \& Wonham 1995).

\subsection{Petri Net models}

Petri Nets (PN) are one of the oldest and most popular modelling frameworks for discrete event dynamic systems. Ever since its introduction in C A Petri's doctoral dissertation (Petri 1962), it has been widely used along with its many variants, for modelling a large variety of systems such as computer software and hardware, distributed database systems, communciation protocols, industrial process control, formal languages, flexible manufacturing systems, etc. The usefulness of this modelling framework is that, apart from answering many logical questions, it can also address issues related to performance of the model. A number of tutorial and survey papers on this topic have appreared recently in the literature (Murata 1989; David \& Alla 1994) along with some books (Peterson 1981).

A Petri Net can be viewed as a modelling tool with both graphical and mathematical features. Graphical nature helps in the visual interpretation of the model, while the mathematical aspect allows easy analysis. This fact, combined with the descriptive power of the nets, makes it a very attractive tool for both practitioners as well as theoreticians.

A Petri Net, in its basic form, is a directed graph with two kinds of nodes, namely, places and transitions. A place is denoted by a circle, while a transition is represented by a rectangular box or a bar. The arcs are directed and connect places to transitions or transitions to places. That is, the structure of the Petri Net is a bipartite graph. See figure 8 for an illustration. The $p_{i}$ '-s denote places while $t_{i}{ }^{\prime}-\mathrm{s}$ stand for the transitions.

To each place is associated a nonnegative variable whose value is equal to the number of marks or tokens at any instant. The number of tokens can correspond to some data or the satisfaction of some conditions associated with the place. A marking $M$ is a state vector that captures the distribution of tokens in all the places in the net. A transition (event) has 


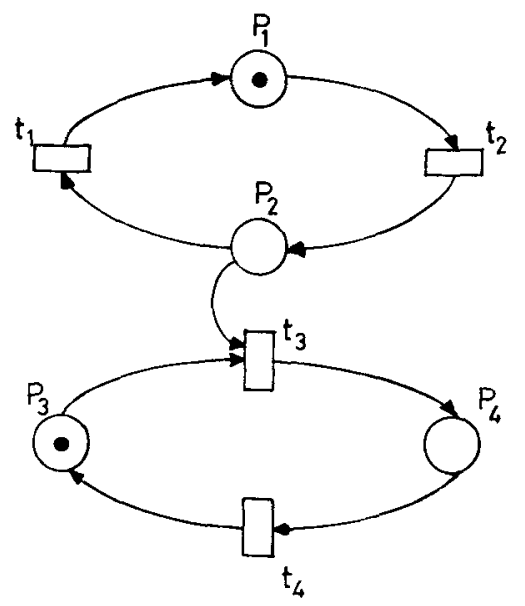

Figure 8. Example of a Petri Net.

a certain number of input places. The token distribution changes, when a certain transition fires, based on the following rules.

(i) A transition can fire only if it is enabled. It is said to be enabled, when each of its input places contains at least one token.

(ii) When a transition fires, each of the input places loses one token, while each of the output places gains one.

Note that, merely the satisfaction of condition (i) does not ensure that the transition will fire.

A major advantage of the PN framework is the associated support for analysis of many properties and problems. Some properties, known as behavioural properties, depend on the initial marking $M_{0}$. Another type of properties depends only on the topological structure. These are known as structural properties.

Among the behavioural properties of Petri Nets, some important ones are described below. For further details the reader is referred to Murata (1989).

- Reachability: A marking $M_{r}$ is said to be reachable from a marking $M_{i}$ if there exists a firing sequence which transforms $M_{i}$ to $M_{r}$. A firing sequence is denoted often as $s \doteq M_{0} t_{n} M_{1} \ldots t_{n} M_{n}$ or simply as $s=t_{1} \ldots t_{n}$. In this case, $M_{n}$ is reachable from $M_{0}$, denoted by $M_{0}\left[s>M_{n}\right.$. The set of all possible markings reachable from $M_{0}$ in a net $N$ is denoted by $R\left(N, M_{0}\right)$ or $R\left(M_{0}\right)$. The set of all possible firing sequences from $M_{0}$ is denoted by $L\left(N, M_{0}\right)$ or $L\left(M_{0}\right)$. The reachability problem is to determine whether $M_{n} \in R\left(M_{0}\right)$ for a given marking $M_{n}$ in a net $\left(N, M_{0}\right)$. It has been shown that this problem is decidable, although of exponential complexity (Murata 1989).

- Liveness: A marking $M_{i}$ is said to be live if, from all markings reachable from it, any transition of the net can be eventually fired, thus ensuring deadlock-free operation. Liveness is often a very strong requirement in many systems. Several levels of liveness have been defined starting from "dead" to L4-live (or live). See Murata (1989) and the references therein for details.

- Boundedness: A marking $M_{i}$ is said to be $l$-bounded, if there exists an integer $l$ such that each place of the net has at most $l$ tokens for every marking reachable from $M_{i}$. In 
particular, a Petri Net is said to be safe if it is 1-bounded. Since, places in a Petri Net are often used to represent buffers and registers, to verify whether it is bounded and safe is an important problem.

- Reversibility: A PN is said to be reversible, if, for each marking $M$ in $R\left(M_{0}\right), M_{0}$ is reachable from $M$. This is therefore some kind of inverse reachability. Often, the reversibility is defined from some home state, rather than the initial state $M_{0}$.

- Persistence: A PN is said to be persistent, if, for any two enabled transitions, the firing of one transition will not disable the other. The concept is closely related to conflict freeness.

- Fairness: Two basic concepts of fairness are the following.

- Bounded fairness. Two transitions $t_{1}$ and $t_{2}$ are said to be in a bounded fair (or $\mathrm{B}$-fair) relation, if the maximum number of times that either one can fire, while the other is not firing, is bounded. A net is said to be B-fair if every pair of transitions is in a B-fair relation.

- Unconditional (global) fairness. A firing sequence $s$ is said to be unconditionally or globally fair, if it is finite or every transition in the net appears infinitely often in $s$. The net is said to be unconditionally fair if every firing sequence has this property.

These properties can be systematically verified by representing the net in the form of an incidence matrix $A=\left[a_{i j}\right]$ which has $n$-rows corresponding to the number of transitions and $m$-columns for the places. It consists of integer elements, and a typical entry is given by

$$
a_{i j}=a_{i j}^{+}+a_{i j}^{-},
$$

where $a_{i j}^{+}$is the number of arcs from transition $i$ to the output place $j$ and $a_{i j}^{-}$is the number of arcs to transition $i$ from the input place $j$. In essence, $a_{i j}^{-}, a_{i j}^{+}$and $a_{i j}$ represent the number of tokens removed, added and changed in place $j$, when transition $i$ fires. Transition $i$ is enabled at marking $M$ if and only if $a_{i j}^{-} \leq M(j), j=1,2, \ldots m$, where $M(j)$ denotes the number of tokens in place $j$ under marking $M$.

Denoting the stage after the $k$ th firing of the net as $M_{k}$, one can write a state equation

$$
M_{k}=M_{k-1}+A^{T} U_{k}, k=1,2, \ldots
$$

where $U_{k}$ is an $n \times 1$ binary column vector containing exactly one non-zero entry (equal to 1 ) in the $i$ th position corresponding to the transition which has fired at the $k$ th instant.

The problems of reachability, liveness and boundedness, are fortunately decidable in this framework, and algorithms exist for their verification (Murata 1989).

Structural properties comprise the following.

- Structural liveness: A PN is said to be structurally live, if there exists a live initial marking.

- Controllability: A net is said to be completely controllable if any marking is reachable from any other marking. It can be shown that if a PN with $m$ places is completely controllable, then we have rank $A=m$ (Murata 1989). For some special classes of PN, this condition is also sufficient. 
- Structural boundedness: A net is said to be structurally bounded, if it is bounded for any finite initial marking $M_{0}$. This property is characterized by the existence of an $m$-vector $y$ of positive integers such that $A y \leq 0$ (Murata 1989). A place $p$ in a PN is strueturally unbounded if there exists a marking $M_{0}$ and a firing sequence $s$ from $M_{0}$ such that $p$ is unbounded.

- Conservativeness: A net is said to be (partially) conservative if there exists a positive integer $y(p)$ for every (some) place $p$ such that the weighted sum of tokens, $M_{y}^{T}=$ $M_{0} y=$ a constant, for every $M \in R\left(M_{0}\right)$ and for any fixed initial marking $M_{0}$. This property can be related to the existence of any $m$-vector $y$ of positive (or negative) integers such that $A y=0, y \neq 0$.

- Repetitiveness: A PN is said to be (partially) repetitive if there exists a marking $M_{0}$ and a firing sequence $s$ from $M_{0}$ such that every (some) transition occurs infinitely often in $s$. This property is satisfied iff then exists an $n$-vector $x$ of positive (nonnegative) integers such that $A^{T} x \geq 0, x \neq 0$.

- Consistency: A net is said to be (partially) consistent if there exists a marking $M_{0}$ and a firing sequence $s$ from $M_{0}$ back to $M_{0}$ such that every (some) transition occurs at least once in $s$. The necessary and sufficient condition for consistency is the existence of an $n$-vector $x$ of positive (nonnegative) integers such that $A^{T} x=0, x \neq 0$.

- $S$ - and T-invariance: An $m$-vector y ( $n$-vector $x$ ) of integers is called an $S$-invariant (T-invariant) if $A y=0\left(A^{T} x=0\right)$.

- Structural B-fairness: This is an extension of the B-fairness property to any initial marking.

There are several abbreviations and extensions of the basic Petri Nets. Abbreviations are simplified graphical representations having one-to-one correspondence with the basic net and, naturally, the same descriptive power. Examples of abbreviations are generalized $\mathrm{PN}$, where positive integral weights are associated to the arcs, coloured $\mathrm{PN}$, in which each token has an identification (colour) and finite capacity PN, where input transitions cannot be fired if that causes a prespecified token holding capacity of an output place to be exceeded.

Among several extensions to the basic PN are priority $\mathrm{PN}$, where there exists a partial order relation on the net transitions and continuous $\mathrm{PN}$, in which the marking of a place is a positive real number and not restricted to be an integer.

All the types of PN discussed above are known as autonomous, which can deal with only logical questions related to the net. Extensions of Petri Nets, which allow one to address timing and performance related issues are known as non-autonomous. Among them Synchronized Petri Net is one where the firing of transitions are synchronized with external events. A transition will be fired if it is enabled and the associated event occurs.

Timed Petri Net is useful for evaluating the performance of a system by associating a timing either with the places (P-timed) or the transitions (T-timed). In P-timed PN, tokens become available at a place $p_{i}$, only after a certain time $d_{i}$ has elapsed after its deposition. Normally, the succeeding transition occurs as soon as all its input places have available tokens, unless some conflict occurs. The T-timed PN is the dual of this. 
Interpreted Petri Nets allow modelling of logical controllers and real-time systems and have the following features:

(i) They are synchronized.

(ii) They are P-timed.

(iii) They have a data-processing feature where the state vectors can be modified in arbitrary ways depending upon the events taking place. A transition can be fired, only if it is enabled and a certain boolean condition associated with it is true.

Stochastic Petri Net is a generalization of T-timed PN, where a random time is associated with the firing of a transition. It is generally assumed that the timings are exponentially distributed. This allows analysis of performance in a probabilistic setting using tools such as Markov Chains, Queueing Theory etc.

The Petri Nets have the advantage that before quantitative evaluation is performed, the qualitative analysis is possible. The disadvantage is that the $\mathrm{PN}$ is not a structured modelling tool, such as some structured languages and Statecharts. However, different approaches have recently been suggested for systematic model building using PN (Ferrarini 1992; Zhou et al 1993; Ausfelder et al 1994; Ferrarini et al 1994).

\subsection{Algebraic models}

The process algebra models aim at describing complex DES in terms of an algebra consisting of a few basic terms, which can be related to some fundamental processes, and a number of operators, which can be used to form complex terms (processes) from simpler ones (Hennessy 1988). The basic terms represent some simple DES behaviour. The different operators arise naturally from the physical fact that simpler DESs indeed operate concurrently or sequentially producing complex behaviours. Such models have been investigated for nearly two decades for providing clear mathematical semantics to programming languages. The developed theory is general enough to model DES dynamics. Among different variants of this technique, the finitely recursive process (FRP) model based on the framework of communicating sequential processes (CSP) has been proposed recently (Inan \& Varaiya 1988)

The central idea in FRP is a process. A process describes the behaviour of a DES in terms of three components: alphabet, trace and termination. Formally speaking, $P=$ $(\alpha P, \operatorname{tr} P, \tau P)$. The alphabet of $\mathrm{P}, \alpha P$, is a set of event symbols, each of which is the abstraction of a physical event which is of concern in the dynamics of the physical process that is intended to be modelled by $P$. The trace of $P$, namely $\operatorname{tr} P$, which is a subset of $(\alpha P)^{*}$, captures the set of event trajectories that can 'take place' in the physical process described by $P$. The termination function $\tau P$ indicates whether a process has terminated successfully or not.

The basic processes of this framework are $S T O P_{A}$ and $S K I P_{A}$. Each of them is a do nothing process, i.e., they generate empty traces. The difference between them is that while the former represents a deadlocked situation or unsuccessful termination, the latter represents a successful termination.

$$
\operatorname{STOP}_{A}:=\left(\operatorname{trSTOP}_{A}:=\{\langle\rangle\}, \alpha \operatorname{STOP}_{A}(\langle\rangle):=A, \tau \operatorname{\tau STOP}_{A}:=0\right)
$$




$$
S K I P_{A}:=\left(\operatorname{trSKIP} A:=\{(\rangle\}, \alpha S K I P_{A}(\langle\rangle):=A, \tau S K I P_{A}:=1\right)
$$

The important operators are as below.

- Deterministic choice operator (DCO): Given $P_{1}, \cdots, P_{n}$ and $\left\{\sigma_{1}, \cdots, \sigma_{n}\right\} \subseteq A, P=$ $\left(\sigma_{1} \rightarrow P_{1}|\cdots| \sigma_{n} \rightarrow P_{n}\right)_{A, \tau}$ is defined as follows:

If $\tau=1$,

$$
P:=S K I P_{A} .
$$

If $\tau=0$ then

$$
\begin{aligned}
\operatorname{tr} P & :=\{\langle\rangle\} \cup\left\{\left\langle\sigma_{i}\right\rangle^{\wedge} s \mid s \in \operatorname{tr} P_{i}, 1 \leq i \leq n\right\}, \\
\alpha P(\langle\rangle) & :=A, \alpha P\left(\left\langle\sigma_{i}\right\rangle^{\wedge} s\right):=\alpha P_{i}(s), \\
\tau P(\langle\rangle) & :=0, \tau P\left(\left\langle\sigma_{i}\right\rangle^{\wedge} s\right):=\tau P_{i}(s) .
\end{aligned}
$$

It says that the initial set of events that can take place in $P$ are from the set $\left\{\sigma_{1}, \cdots, \sigma_{n}\right\}$ and after occurrence of $\sigma_{i}$ the process $P$ starts behaving as $P_{i}$. As an example, consider the process $P=(a \rightarrow P \mid b \rightarrow S K I P\{\})_{\{a, b\}, 0}$ which generates the language $\operatorname{tr} P=$

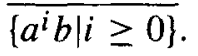

- Sequential composition operator (SCO): Given $P_{1}$ and $P_{2}, P_{1} ; P_{2}$ is defined as follows:

$$
\begin{aligned}
& \alpha\left(P_{1} ; P_{2}\right)(\langle\rangle):=\left(\alpha P_{1}(\langle\rangle) \text { if } \tau P_{1}(\langle\rangle)=0\right) \vee\left(\alpha P_{2}(\langle\rangle)\right. \\
& \text { if } \left.\tau P_{1}(\langle\rangle)=1\right) \text {, } \\
& \tau\left(P_{1} ; P_{2}\right)(\langle\rangle):=\left(0 \quad \text { if } \tau P_{1}(\langle\rangle)=0\right) \vee\left(\tau P_{2}\left(\langle) \quad \text { if } \tau P_{1}(\langle\rangle)=1\right)\right. \text {, } \\
& \left(P_{1} ; P_{2}\right) /\langle\sigma\rangle:=\left\{\begin{array}{l}
\left(P_{1} /\langle\sigma\rangle\right) ; P_{2}, \quad \text { if }\langle\sigma\rangle \in \operatorname{tr} P_{1}, \\
P_{2} /\langle\sigma\rangle, \text { if } \tau P_{1}(\langle\rangle)=1 \wedge\langle\sigma\rangle \in \operatorname{tr} P_{2}, \\
\text { undefined, otherwise. }
\end{array}\right.
\end{aligned}
$$

Here $P$ initially behaves as $P_{1}$ and after $P_{1}$ terminates successfully, it behaves as $P_{2}$. Consider the processes $Q=\left(a \rightarrow Q ; R \mid b \rightarrow \operatorname{SKIP}_{\{\}}\right)_{\{a, b\}, 0}$ and $R=(a \rightarrow$ $S K I P\{\})_{\{a\}, 0}$. Then $\operatorname{tr} Q=\overline{\left\{a^{i} b a^{i} \mid i \geq 0\right\}}$.

- Parallel composition operator (PCO): Given $P_{1}$ and $P_{2}, P_{1} \| P_{2}$ is defined as follows: $\alpha\left(P_{1} \| P_{2}\right)(\langle\rangle):=\alpha P_{1}(\langle\rangle) \cup \alpha P_{2}(\langle\rangle)$.

$$
\begin{aligned}
& \tau\left(P_{1} \| P_{2}\right)(\langle):= \\
& \left\{\begin{array}{l}
1, \quad \text { if }\left\{\begin{array}{l}
\left(\tau P_{1}(\langle\rangle)=1 \wedge \tau P_{2}(\langle\rangle)=1\right) \vee \\
\left(\tau P_{1}(\langle\rangle)=1 \wedge \alpha P_{2}(\langle\rangle) \subseteq \alpha P_{1}(\langle))\right) \vee \\
\left(\tau P_{2}(\langle\rangle)=1 \wedge \alpha P_{1}(\langle)) \subseteq \alpha P_{2}(\langle\rangle)\right),
\end{array}\right. \\
0, \quad \text { otherwise. }
\end{array}\right. \\
& \left(P_{1} \| P_{2}\right) /(\langle\sigma\rangle):= \\
& \begin{cases}\left(P_{1} /\langle\sigma\rangle\right) \| P_{2}, & \text { if }\langle\sigma\rangle \in \operatorname{tr} P_{1} \wedge \sigma \notin \alpha P_{2}(\langle\rangle), \\
\left(P_{2} /\langle\sigma\rangle\right) \| P_{1}, & \text { if }\langle\sigma\rangle \in \operatorname{tr} P_{2} \wedge \sigma \notin \alpha P_{1}(\langle\rangle), \\
\left(P_{1} /\langle\sigma\rangle \|\left(P_{2} /\langle\sigma\rangle\right),\right. & \text { if }\langle\sigma\rangle \in \operatorname{tr} P_{1} \cap \operatorname{tr} P_{2}, \\
\text { undefined, } & \text { otherwise. }\end{cases}
\end{aligned}
$$


It represents the concurrent behaviour of $P_{1}$ and $P_{2}$. Any arbitrary interleaving of event sequences from $P_{1}$ and $P_{2}$ can take place in $P$ except that any event belonging to their common alphabet must take place synchronously in both components, and it will be shown as a single event in the trace of $P$.

Due to variable alphabet, unlike that of CSP, there normally does not exist a global set of synchronised events in parallel composition. However using the global change operator, constant synchronizations can be modelled. Consider the processes $S=$ $\left(a \rightarrow S \mid b \rightarrow S K I P_{\{\}}\right)_{\{a, b\}, 0}$ and $T=\left(a \rightarrow S K I P_{\{b\}}\right)_{\{a\}, 0}$. Then $\operatorname{tr}(S \| T)=$ $\overline{\left\{a^{i} \mid i \geq 0\right\} \cup\{b a\}}$.

- Local change operator ( $L C O$ ): Given a process $P$ and collection of events $B$ and $C$, the $\operatorname{LCO} P^{[-B+C]}$ is defined as follows.

$$
\begin{aligned}
\alpha P^{[-B+C]}(\langle\rangle) & :=(\alpha P(\langle\rangle)-B) \cup C, \\
\tau P^{[-B+C]}(\langle\rangle) & :=\tau P(\langle\rangle), \\
P^{[-B+C]} /\langle\sigma\rangle & := \begin{cases}(P /\langle\sigma\rangle, & \text { if }\langle\sigma\rangle \in \operatorname{tr} P \wedge \sigma \notin B), \\
\text { undefined, } & \text { otherwise. }\end{cases}
\end{aligned}
$$

As an example, note that, for the process $P$ used in the example of $D C O, \operatorname{tr} P^{[-\{b\}+\{c\}]}=$ $\left\{a^{i} \mid i \geq 0\right\}$.

- Global change operator (GCO): Given a process $P$ and collection of events $B$ and $C$, the GCO $P^{[[-B+C]]}$ is defined as follows.

$$
\begin{aligned}
\alpha P^{[-B+C]]}(\langle\rangle) & :=(\alpha P(\langle\rangle)-B) \cup C, \\
\tau P^{[[-B+C]]}(\langle\rangle) & :=\tau P(\langle\rangle), \\
P^{[[-B+C]]} /\langle\sigma\rangle & := \begin{cases}(P /\langle\sigma\rangle)^{[-B+C]]}, & \text { if }\langle\sigma\rangle \in \operatorname{tr} P \wedge \sigma \in / B), \\
\text { undefined. } & \text { otherwise. }\end{cases}
\end{aligned}
$$

Again note that, for the process $P$ used in the example of $D C O, P^{[[-\{b\}+\{c\}]]}=Z=$ $(a \rightarrow Z)_{\{a, c\}, 0}$

A process $Y$ is said to be a Finitely Recursive Process (FRP) if it can be represented as:

$$
\mathbf{X}=\mathbf{F}(\mathbf{X}), Y=g(\mathbf{X})
$$

where $\mathbf{X}=\left(X_{1}, \ldots, X_{n}\right)$, a vector of processes, $\mathbf{X}$ is of the form

$$
F_{i}(\mathbf{X})=\left(\sigma_{i_{1}} \rightarrow f_{i_{1}}(\mathbf{X})|\cdots| \sigma_{i_{k_{i}}} \rightarrow f_{i_{k_{i}}}(\mathbf{X})\right)_{\alpha_{i}, \tau_{i}}
$$

and $f_{i_{j}}$ and $g$ are composed of the operators PCO, SCO, LCO and GCO. In the example section we have shown how to model the dynamics of a manufacturing cell using such recursive equations.

The framework in this form lacks certain modelling flexibilities such as absence of timing features, data variables etc. Recently this has been extended to incorporate these features (Bose \& Mukhopadhyay 1995). A nondeterministic extension has also been proposed (Bose et al 1995b). Unfortunately, certain key properties like boundedness, reachability etc., become undecidable in this framework. This arises because of unrestricted use of both parallel and sequential composition operators. For maintaining tractability, it is 
necessary to restrict them in some fashion so that one can find computational algorithms for solving specific problems. Some initial work in this direction has been reported (Bose et al 1995a, c).

\section{An example}

In this section we consider an automated manufacturing system taken from Zhou et al (1992) for modelling using the frameworks of FRP, Statechart and TTM. A Petri Net model, already given by Zhou et al (1992), is presented for comparison. An FSM model is shown to have too large a state space to be described here.

The layout of the automated manufacturing plant is shown in figure 9. It consists of four machines, two robots, two buffers of capacity $b_{1}$ and $b_{2}$ respectively and an assembly cell. The system first processes two types of parts, called $F$ and $G$ from common raw materials, and then assembles these parts pair by pair. An $F$-part is first processed by machine 1, then it goes to buffer 1 , and is finally processed by machine 3. Machine unloading and transfer operations are conducted with the help of robot 1 . The same generic procedure is used to produce a $G$ part as shown on the right side of figure 9, i.e., machine 2, buffer 2 , machine 4 and robot 2 for unloading and transfer. The system requires that raw material

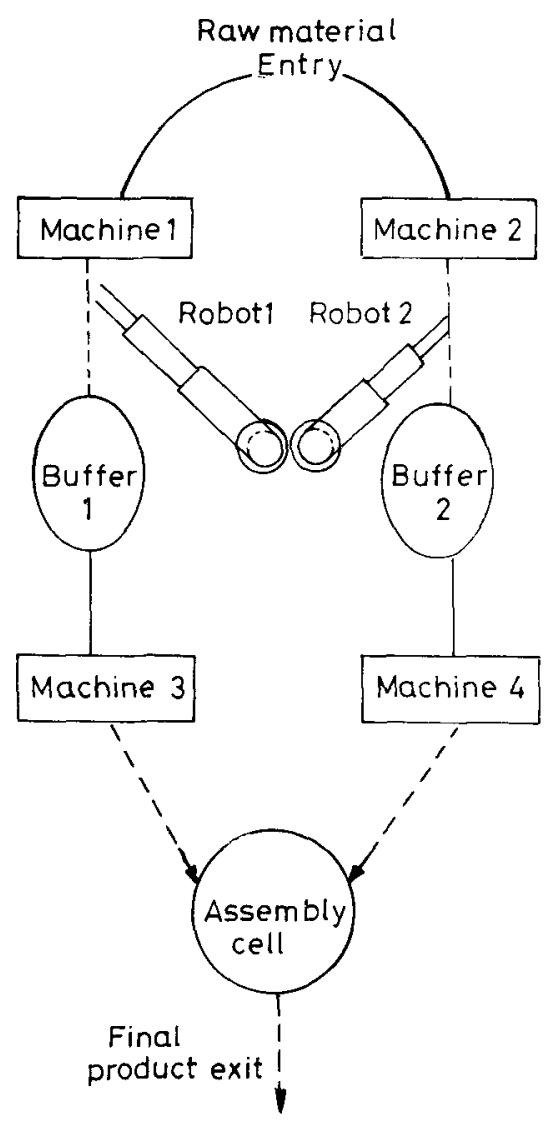

Figure 9. Layout of an automated manufacturing plant. 


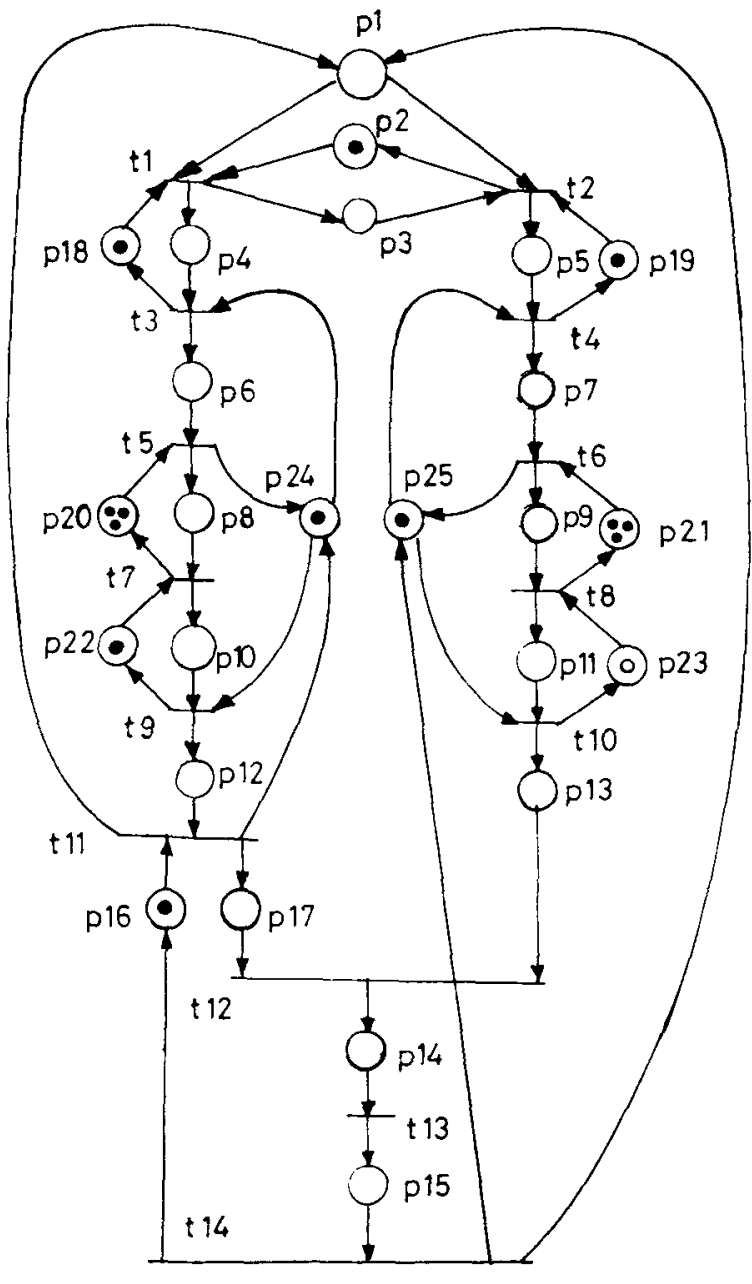

Figure 10. Petri Net model of the plant.

for $F$-part will go to the machine 1 first, and then raw material for $G$-part goes to the 2 nd machine. Finally at the assembly stage, an $F$-part is required to enter the assembly station first, followed by a $G$-part. Robot 2 is responsible for the assembly process and delivery of the final product to the output area.

\subsection{PN model}

The PN model is taken from Zhou et al (1992) and is shown in figure 10. In the figure, $m_{0}\left(p_{i}\right)$ denotes the initial marking of the place $p_{i}$. If unspecified, it is assumed to be zero. Note how the places correspond to the various states of activity. When a token is available in a place, the corresponding activity occurs. Since many places can simultaneously contain a token, those activities go on concurrently. The transitions represent start or end of operation, which cause changes of state. The places $p_{1}, p_{2}, p_{3}$ model the states of the controlling mechanism to ensure that $t_{1}$ fires first and then $t_{2}$ and $t_{1}$ fire alternately. Places $p_{4}, p_{6}$, $p_{8}, p_{10}, p_{12}, p_{18}, p_{20}, p_{22}$ along with the related transitions model the production of an 
$F$-part. A similar structure corresponds to the $G$-part. The assembly cell is modelled by the places $p_{14}$ to $p_{17}$ along with transitions $t_{12}$ to $t_{14}$. The various types of logical analysis that can be done using the developed model have been discussed (Zhou et al 1992).

\subsection{FRP model}

While modelling the manufacturing cell using an FRP, we adopt a modular approach. Each of the machines, buffers, and robots, the raw material entry point and the assembly cell is modelled as a process. The overall system consists of these component subsystems evolving concurrently and is modelled by a PCO. The overall sequence is achieved by interleaving, with synchronization and blocking among events from the different subsystems. The names of the events used in the model are self-explanatory.

- Machine-1:

$$
\begin{aligned}
& M_{-} 1=\left(\text { load }_{-} F \rightarrow M_{--} 1^{\prime}\right)_{\left\{l o a d_{-} F\right\}, 0} \\
& M_{-} 1^{\prime}=\left(\text { tr } F_{-} B_{-} \text {pick } \rightarrow M_{-} 1\right)_{\left\{t r_{-} F_{-} B_{-} p i c k\right\}, 0} \\
& \cdot \text { Machine-2: } \\
& M_{-} 2=\left(\text { load } G \rightarrow M_{-} 2^{\prime}\right)_{\left\{l o a d_{-} G\right\}, 0} \\
& M_{-} 2^{\prime}=\left(\text { tr } G_{-} B_{-} \text {pick } \rightarrow M_{-} 2\right)_{\left\{t r_{-} G_{-} B_{-} \text {pick }\right\}, 0} \\
& \cdot \text { Raw material entry point : } \\
& R M=\left(\text { load_F } \rightarrow R M^{\prime}\right)_{\left\{l o a d_{-} F\right\}, 0} \\
& R M^{\prime}=(\text { load } G \rightarrow R M)_{\left\{l o a d_{-} G\right\}, 0}
\end{aligned}
$$

The $R M$ process, by synchronization, ensures that an $F$ part in $M 1$ and a $G$ part in $M 2$ are processed alternately.

\section{- Buffer-1 :}

$$
\begin{aligned}
& B 1_{-} E=\left(t r_{-} F_{-} B_{-} d r o p \rightarrow B 1_{-} 1\right)_{\left\{t r_{-} F_{-} B_{-} d r o p\right\}, 0} \\
& B 1_{-} 1=\left(t_{-} F_{-} B_{-} d r o p \rightarrow B 1_{-} 2\right. \\
& \left.\left.\mid \text { load_L }_{-} 3 \rightarrow B 1_{-} E\right)_{\left\{t r_{-} F_{-} B_{-}\right. \text {drop, load_M }} M_{-}\right\}, 0 \\
& B 1_{-} \overline{b_{1}-1}=\left(t r_{-} F_{-} B_{-} d r o p \rightarrow B 1_{-} F\right. \\
& \text { [ load-M_3 } \left.\rightarrow B 1_{-} \overline{b_{1}-2}\right)_{\left\{t r_{-} F_{-} B_{-} \text {drop load_- }_{-} 3\right\}, 0} \\
& B 1_{-} F=\left(\text { load }_{-} M_{-} 3 \rightarrow B 1_{-} \overline{b_{1}-1}\right)_{\left\{l o a d_{-} M_{-} 3\right\}, 0}^{\left[+\left\{t r_{-} F_{-} B_{-} \text {pick }\right\}\right]} \\
& \text { - Buffer-2 : } \\
& B 2_{-} E=\left(t r_{-} G_{-} B_{-} d r o p \rightarrow B 2_{-} 1\right)_{\left\{t r_{-} G_{-} B_{-} d r o p\right\}, 0} \\
& B 2-1=\left(t r_{-} G_{-} B_{-} d r o p \rightarrow B 22_{-}\right. \\
& \left.\mid \text {load_M_4 } \rightarrow B 2_{-} E\right)_{\left\{t r_{-} G_{-} B_{-} d r o p, l o a d_{-} M_{-} 4\right\}, 0} \\
& B 2 \_\overline{b_{2}-1}=\left(t r_{-} G_{-} B_{-} d r o p \rightarrow B 2_{-} F\right. \\
& \text { | } \left.\text { load }_{-} M_{-} 4 \rightarrow B 2-\overline{b_{2}-2}\right)_{\left\{t r_{-} G_{-} B_{-} \text {drop,load }-M_{-} 4\right\}, 0} \\
& B 2_{-} F=\left(l_{\text {load }} M_{-} 4 \rightarrow B 2_{-} \overline{b_{2}-1}\right)_{\left\{l o a d_{-} M_{-} 4\right\}, 0}^{\left[+\left\{t G_{-} B_{-} \text {pick }\right\}\right]}
\end{aligned}
$$

Note that, due to lack of variables, for each buffer state a process is defined and if the buffer capacity is large, the model becomes cumbersome. Also it is to be noted that robots are prevented from picking up objects from machines when the respective buffers are full 
and this has been achieved using an LCO.

- Machine-3:

$M_{-} 3=\left(\operatorname{load}_{-} M_{-} 3 \rightarrow M_{-} 3^{\prime}\right)_{\left\{l o a d_{-} M_{-} 3\right\}, 0}$

$M_{-} 3^{\prime}=\left(t r_{-} F_{-} A_{-} p i c k \rightarrow M_{-} 3\right)_{\left\{t r_{-} F_{-} A_{-} p i c k\right\}, 0}$

- Machine-4:

$M_{-} 4=\left(\operatorname{load}_{-} M_{-} 4 \rightarrow M_{-} 4^{\prime}\right)_{\left\{l o a d_{-} M_{-} 4\right\}, 0}$

$M_{-} 4^{\prime}=\left(t r_{-} G_{-} A_{-} p i c k \rightarrow M_{-} 4\right)_{\left\{t r_{-} G_{-} A_{-} p i c k\right\}, 0}$

Machines 3 and 4 are similar to the machines 1 and 2 respectively.

- Assembly cell:

$A C_{-} E=\left(t r_{-} F_{-} A_{-} d r o p \rightarrow A C_{-} F\right)_{\left\{t r_{-} F_{-} A_{-} d r o p\right\}, 0}$

$A C_{-} F=\left(t r_{-} G_{-} A_{-} d r o p \rightarrow A C_{-} F G\right)_{\left\{t r_{-} G_{-} A_{-} \text {drop }\right\}, 0}^{\left[+\left\{r_{-} F_{-} A_{-} p i c k\right]\right.}$

$A C_{-} F G=\left(s t_{-} a s s m \rightarrow A C_{-} W\right)_{\left\{s t_{-} a s s m\right\}, 0}^{\left[+\left\{r_{-} F_{-} A_{-} p i c k, t r_{-} G_{-} A_{-} p i c k\right\}\right]}$

$A C_{-} W=\left(\text { fin_assm } \rightarrow A C_{-} E\right)_{[\text {fin }}^{\left[+\left\{t r_{-} F_{-} A_{-} p i c k\right\}, 0\right.}$

In the assembly cell process, again, using LCO, robot 1 is prevented from picking up a part from $M 3$ when the assembly cell is not empty. Similarly robot 2 cannot pick another part from $M 4$ after it has dropped one $G$-part in the assembly cell till the assembly operation is completed. Otherwise a deadlocked situation will arise.

- Robot-1:

$$
\begin{aligned}
R_{-} 1_{-} I= & \left(t r_{-} F_{-} B_{-} \text {pick } \rightarrow R_{-} 1_{-} W 1\right. \\
& \left.\mid t r_{-} F_{-} A_{-} \text {pick } \rightarrow R_{-} 1_{-} W 2\right)_{\left\{t r_{-} F_{-} B_{-} p i c k, t r_{-} F_{-} A_{-} p i c k\right\}, 0} \\
R_{-} 1_{-} W 1=\left(t r_{-} F_{-} B_{-} d r o p\right. & \left.\rightarrow R_{-} 1-I\right)_{\left\{t r_{-} F_{-} B_{-} d r o p\right\}, 0} \\
R_{-} 1_{-} W 2=\left(t r_{-} F_{-} A_{-} d r o p\right. & \left.\rightarrow R_{-} 1_{-} I\right)_{\left\{t r_{-} F_{-} A_{-} d r o p\right\}, 0}
\end{aligned}
$$

- Robot-2:

$R_{-} 2_{-} I=\left(t r_{-} G_{-} B_{-} p i c k \rightarrow R_{-} 2_{-} W 1 \mid t r_{-} G_{-} A_{-} p i c k \rightarrow R_{-} 2_{-} W 2\right.$

| st_assm $\left.\rightarrow R_{-} 2_{-} W 3\right)_{\left\{t r_{-} G_{-} B_{-} p i c k, t r_{-} G_{-} A_{-} \text {pick,sta assm\},0 }\right.}$

$R_{-} 2_{-} W 1=\left(t r_{-} G_{-} B_{-} d r o p \rightarrow R_{-} 2_{-} I\right)_{\left\{t r_{-} G_{-} B_{-} d r o p\right\}, 0}$

$R_{-} 2_{-} W 2=\left(t r_{-} G_{-} A_{-} d r o p \rightarrow R_{-} 2_{-} I\right)_{\left\{t r_{-} G_{-} A_{-} d r o p\right\}, 0}$

$R_{-} 2_{-} W 3=\left(\text { fin_assm } \rightarrow R_{-} 2_{-} I\right)_{\{\text {fin_assm }\}, 0}$

Robot 1 has two working states, representing the transfer of parts from machine to buffer and machine to assembly cell. Robot 2 has an extra working state representing the assembly of $F$ and $G$ parts in the assembly.

Also define

$$
\begin{aligned}
& A_{M C_{-} 1}=\left\{l o a d_{-} F, t r_{-} F_{-} B_{-} \text {pick }\right\} \\
& A_{M C_{-} 2}=\left\{l o a d_{-} G, t r_{-} G_{-} B_{-} \text {pick }\right\} \\
& A_{M C_{-} 3}=\left\{l o a d_{-} M_{-} 3, t r_{-} F_{-} A_{-} p i c k\right\} \\
& A_{M C_{-} 4}=\left\{\text { load_M-4,tr-G } \text { lo }_{-} A_{-} \text {pick }\right\} \\
& A_{B_{-} 1}=\left\{l o a d_{-} M_{-} 3, t r_{-} F_{-} B_{-} d r o p\right\} \\
& A_{B_{-} 2}=\left\{l o a d_{-} M_{-} 4, t r_{-} G_{-} B_{-} d r o p\right\} \\
& A_{R_{-}}=\left\{t r_{-} F_{-} B_{-} p i c k, t r_{-} F_{-} B_{-} d r o p, t r_{-} F_{-} A_{-} p i c k, t r_{-} F_{-} A_{-} d r o p\right\} \\
& A_{R_{-} 2}=\left\{t r_{-} G_{-} B_{-} p i c k, t r_{-} G_{-} B_{-} d r o p, t r_{-} G_{-} A_{-} p i c k, t r_{-} G_{-} A_{-} d r o p,\right. \\
& \text { st_assm, fin_assm }\} \\
& A_{R M}=\left\{l o a d_{-} F, l o a d_{-} G\right\} \\
& A_{A C}=\left\{t r_{-} F_{-} A_{-} d r o p, t r_{-} G_{-} A_{-} d r o p, s t_{-} \text {assm, fin_assm }\right\}
\end{aligned}
$$


Now the dynamics of the overall manufacturing cell is given as

M.Cell $=\left(M_{-} 1\right)^{\left[\left[+A_{M C_{-}}\right]\right]}\left\|\left(M_{-} 2\right)^{\left[\left[+A_{M C_{-}} l\right]\right.}\right\|\left(M_{-} 3\right)^{\left[\left[+A_{M C_{-}}\right]\right]} \|$ $\left(M_{-} 4\right)^{\left[\left[+A_{M C_{-}}\right]\right]}\left\|\left(B_{-} 1_{-} E\right)^{\left[\left[+A_{B_{-}}\right]\right]}\right\|\left(B_{-} 2_{-} E\right)^{\left[\left[+A_{B_{-} 2}\right]\right]}\left\|\left(R_{-} 1_{-} I\right)^{\left[\left[+A_{R_{-1}}\right]\right]}\right\|$ $\left(R_{-} 2_{-} l\right)^{\left[\left[+A_{\left.\left.R_{-} 2\right]\right]}\right.\right.}\left\|(R M)^{\left[\left[+A_{R M} \mathrm{l}\right]\right.}\right\|\left(A C_{-} E\right)^{\left[\left[+A_{A C}\right]\right]}$.

Note the use of GCO in achieving a constant synchronizing alphabet while keeping the core process description modular and independent.

\subsection{SC model}

The same set of event symbols that are used in FRP, can be used in SC Model also. The formalism, being a graphical one is shown in figure 11. In table 1 we present the list of events and associated event conditions. The event symbols shown in the table are used in the figure.

Note that the earlier problem of modelling of buffers with large capacity remains, perhaps more so in a graphic environment. Otherwise the graphical nature and the event conditions based on active state of various subcomponents renders modelling easy. However, providing the graphical support is a problem in itself. Moreover there is no clue as to how the event conditions will be verified in an actual physical implementation. Thus this description is at a somewhat higher level than that of FRPs or TTMs.

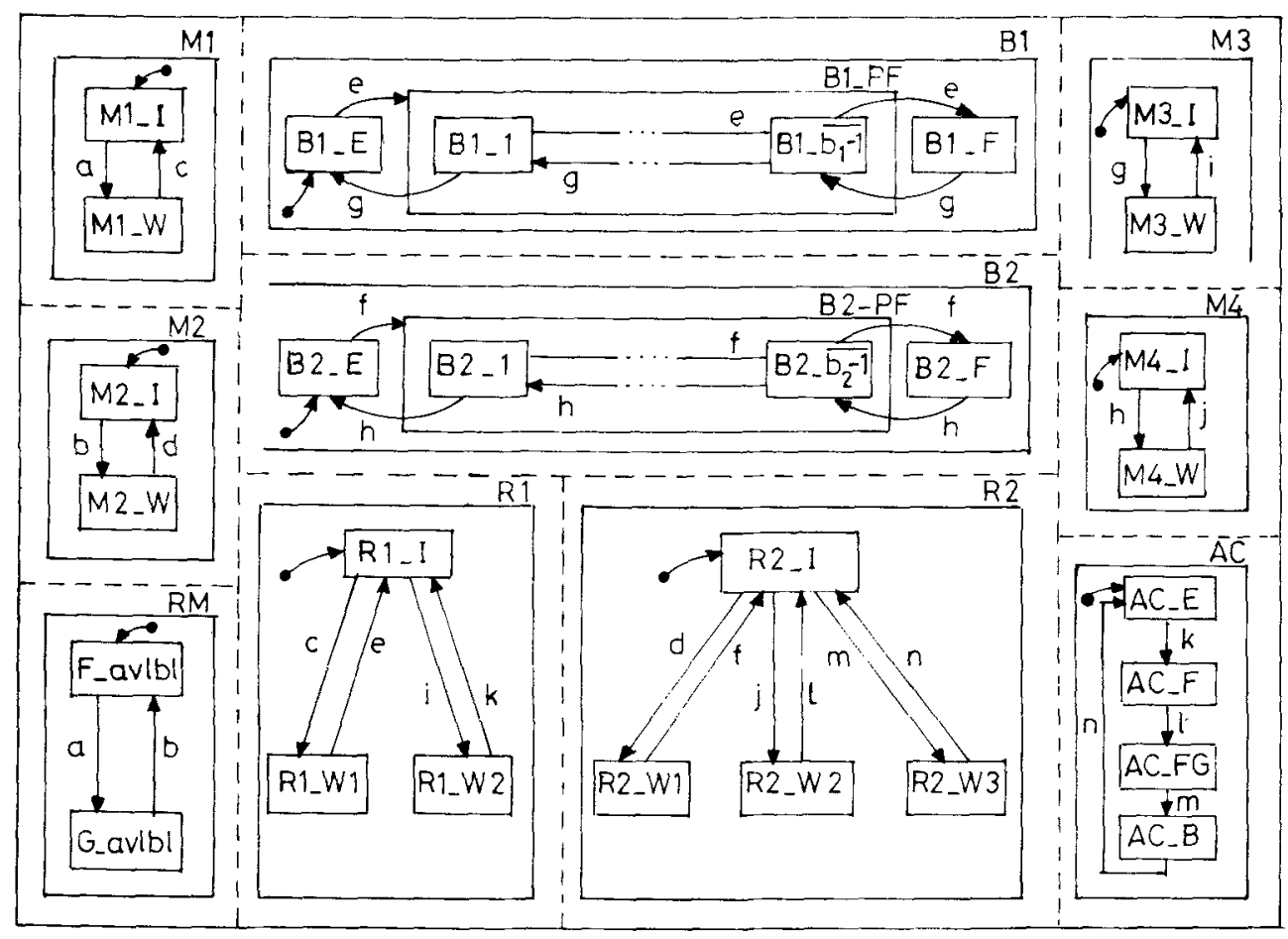

Figure 11. Statechart model of the plant. 
Table 1. Event names, symbols and associated conditions for the statechart model.

\begin{tabular}{|c|c|c|}
\hline Event name & Event condition & Event symbol \\
\hline$l o a d_{-} F$ & $F_{-a} a v l b l$ & $\mathbf{a}$ \\
\hline $\operatorname{load}_{-} G$ & $G_{-} a v l b l$ & b \\
\hline$t r_{-} F_{-} B_{-} p i c k$ & $R 1_{-} I \wedge M 1_{-} W \wedge \sim B 1_{-} F$ & $\mathrm{c}$ \\
\hline$t r_{-} G_{-} B_{-} p i c k$ & $R 2_{-} I \wedge M 2_{-} W \wedge \sim B 2_{-} F$ & $\mathrm{~d}$ \\
\hline$t r_{-} F_{-} B_{-} d r o p$ & $R 1_{-} W 1$ & $\mathrm{e}$ \\
\hline$t r_{-} G_{-} B_{-} d r o p$ & $R 2 \_W 1$ & $f$ \\
\hline$l_{o a d} M_{-} 3$ & $\left(\sim B 1_{-} E\right) \wedge M 3_{-} I$ & $\mathbf{g}$ \\
\hline$l_{\text {load }} M_{-} 4$ & $\left(\sim B 2_{-} E\right) \wedge M 4_{-} I$ & $\mathrm{~h}$ \\
\hline$t r_{-} F_{-} A_{-} p i c k$ & $R 1_{-} I \wedge M 3_{-} W \wedge A C_{-} E$ & $\mathrm{i}$ \\
\hline$t r_{-} G_{-} A_{-} p i c k$ & $R 2_{-} I \wedge M 4_{-} W \wedge A C_{-} F$ & $\mathrm{j}$ \\
\hline$t r_{-} F_{-} A_{-} d r o p$ & $R 1_{-} W 2$ & $\mathbf{k}$ \\
\hline$t r_{-} G_{-} A_{-} d r o p$ & $R 2-W 2$ & 1 \\
\hline st_assm & $R 2_{-} I \wedge A C_{-} F G$ & m \\
\hline fin_assm & true & $\mathbf{n}$ \\
\hline
\end{tabular}

\section{$4.4 \quad$ TTM model}

The TTM model is shown in figure 12. Here again the same modular approach as that of FRP and SC is used. All components are modelled individually as TTMs and the overall system is the parallel composition of individual TTMs. Even though TTM is able to model real time constraints, in the present example we have ignored the real time behaviour of the system and $l_{\tau}$ and $u_{\tau}$ have been chosen as 0 and $\infty$ respectively for all transitions. The initial and tick transitions are common to all the components. The initial transition does not change any state and just initiates the event sequence of a TTM. And tick is the only transition that increments the global clock variable by one unit. Same event symbols as given in the table of statechart are used. However we also used two pairs of communications and two more transitions, namely Check_c1 (symbolo) and Check_c2 (symbol $p$ ).

- Raw material entry point (RM):

$\mathcal{V}_{R M}=\left\{x_{R M}, \eta, t\right\}$

$\mathcal{T}_{R M}=\{$ load_F, load_G, initial, tick $\}$

$\Theta_{R M}=\left(\eta=\right.$ initial $\left.\wedge x_{R M}=F_{a v l b l}\right)$

\begin{tabular}{l|c|c|}
\hline$\tau$ & $e_{\tau}$ & $h_{\tau}$ \\
\hline load_F & $x_{R M}=F_{a v l b l}$ & $x_{R M}:=G_{a v l b l}$ \\
\hline load_G & $x_{R M}=G_{\text {avlbl }}$ & $x_{R M}:=F_{a v l b l}$ \\
\hline
\end{tabular}



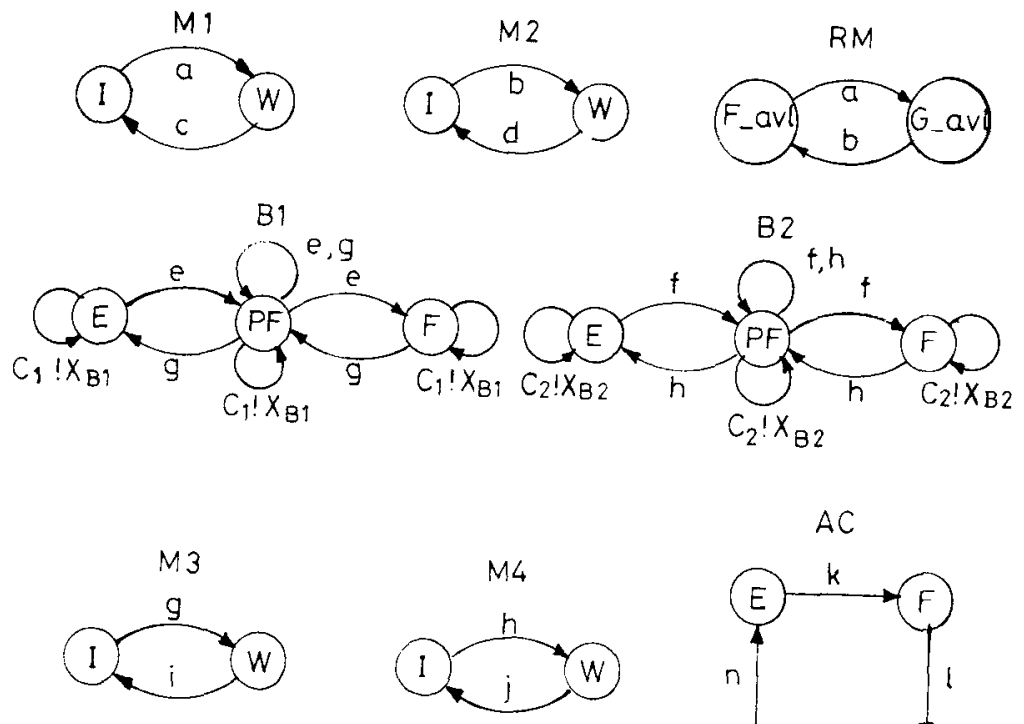

$A C$

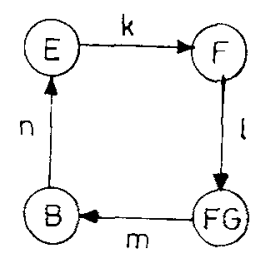

R 1
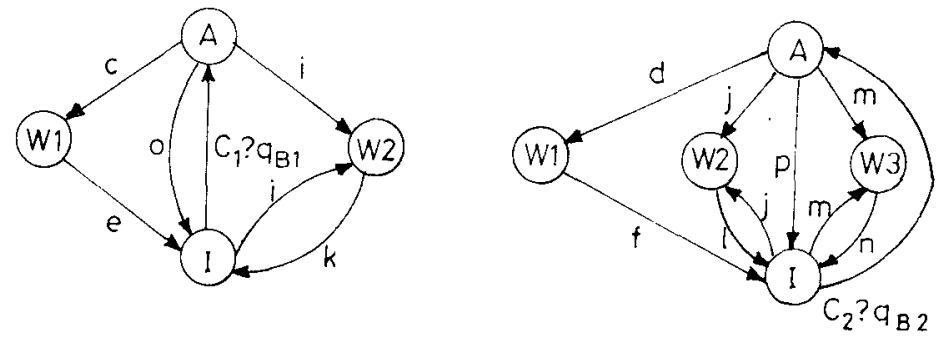

Figure 12. Timed transition model of the plant.

$\Theta_{M 1}=\left(\eta=\right.$ initial $\left.\wedge x_{M 1}=I\right)$

\begin{tabular}{|c|c|c|}
\hline$\tau$ & $e_{\tau}$ & $h_{\tau}$ \\
\hline load_F & $x_{M 1}=I$ & $x_{M 1}:=W$ \\
\hline$t r_{-} F_{-} B_{-}$pick & $x_{M 1}=W$ & $x_{M 1}:=I$ \\
\hline
\end{tabular}

- Machine-2 (M2):

$\mathcal{V}_{M 2}=\left\{x_{M 2}, \eta, t\right\}$

$\mathcal{T}_{M 2}=\left\{l o a d_{-} G, t r_{-} G_{-} B_{-}\right.$pick, initial, tick $\}$

$\Theta_{M 2}=\left(\eta=\right.$ initial $\left.\wedge x_{M 2}=I\right)$

\begin{tabular}{|c|c|c|}
\hline$\tau$ & $e_{\tau}$ & $h_{\tau}$ \\
\hline load_G & $x_{M 2}=I$ & $x_{M 2}:=W$ \\
\hline tro-G_B_pick & $x_{M 2}=W$ & $x_{M 2}:=I$ \\
\hline
\end{tabular}


- Buffer-1 (B1):

$\mathcal{V}_{B 1}=\left\{x_{B 1}, y, \eta, t\right\}$

$\mathcal{T}_{B 1}=\left\{\right.$ load $-M 3, t r_{-} F_{-} B_{-} d r o p, c 1 ! x_{B 1}$, initial, tick $\}$

$\Theta_{B 1}=\left(\eta=\right.$ initial $\left.\wedge x_{B 1}=E \wedge y=0\right)$

\begin{tabular}{|c|c|c|}
\hline$\tau$ & $e_{\tau}$ & $\bar{h}_{\tau}$ \\
\hline$c 1 ! x_{B 1}$ & true & [] \\
\hline load_M3 & $\sim\left(x_{B 1}=E\right)$ & $\begin{array}{c}y:=y-1 \\
\left(1 \leq y<b_{1}\right) \Rightarrow x_{B 1}:=P F \\
(y=0) \Rightarrow x_{B 1}:=E\end{array}$ \\
\hline$t r_{-} F_{-} B_{-} d r o p$ & $\sim\left(x_{B 1}=F\right)$ & $\begin{array}{c}y:=y+1 \\
\left(1 \leq y<b_{1}\right) \Rightarrow x_{B 1}:=P F \\
\left(y=b_{1}\right) \Rightarrow x_{B 1}:=F\end{array}$ \\
\hline
\end{tabular}

- Buffer-2 (B2):

$\nu_{B 2}=\left\{x_{B 2}, z, \eta, t\right\}$

$\mathcal{T}_{B 2}=\left\{\right.$ load_M4,tr-G $G_{-} B_{-}$drop, $c 2 ! x_{B 2}$, initial, tick $\}$

$\Theta_{B 2}=\left(\eta=\right.$ initial $\left.\wedge x_{B 2}=E \wedge z=0\right)$

\begin{tabular}{|c|c|c|}
\hline$\tau$ & $e_{\tau}$ & $h_{\tau}$ \\
\hline$c 2 ! x_{B 2}$ & true & {[]} \\
\hline & & $z:=z-1 ;$ \\
& $\sim\left(x_{B 2}=E\right)$ & $\left(1 \leq z<b_{2}\right) \Rightarrow x_{B 2}:=P F ;$ \\
& & $(z=0) \Rightarrow x_{B 1}:=E$ \\
\hline$r_{-} G_{-} B_{-} d r o p$ & $\sim\left(x_{B 2}=F\right)$ & $\left(1 \leq z<b_{1}\right) \Rightarrow x_{B 2}:=P F ;$ \\
& & $\left(z=b_{2}\right) \Rightarrow x_{B 2}:=F$ \\
\hline
\end{tabular}

The important features to be noted here are : (i) use of data variables in buffer definitions which results in a compact description; (ii) use of communication channels, through which buffers are always ready to communicate their states, and (iii) piecewise constant nature of transformation functions defined in the "Buffer" TTMs.

- Machine-3 (M3):

$\mathcal{V}_{M 3}=\left\{x_{M 3}, \eta, t\right\}$

$\mathcal{T}_{M 3}=\left\{l o a d_{-} M 3, t r_{-} F_{-} A_{-}\right.$pick, initial, tick $\}$

$\Theta_{M 3}=\left(\eta=\right.$ initial $\left.\wedge x_{M 3}=I\right)$

\begin{tabular}{|c|c|c|}
\hline$\tau$ & $e_{\tau}$ & $h_{\tau}$ \\
\hline load_M3 & $x_{M 3}=I$ & $x_{M 3}:=W$ \\
\hline$t r_{-} F_{-} A_{-}$pick & $x_{M 3}=W$ & $x_{M 3}:=I$ \\
\hline
\end{tabular}


- Machine-4 (M4):

$\mathcal{V}_{M 4}=\left\{x_{M 4}, \eta, t\right\}$

$\mathcal{T}_{M 4}=\left\{\right.$ load $-M 4$, tr $\boldsymbol{G}_{-} \boldsymbol{G}_{-} A_{-}$pick, initial, tick $\}$

$\Theta_{M 4}=\left(\eta=\right.$ initial $\left.\wedge x_{M 4}=I\right)$

\begin{tabular}{|c|c|c|}
\hline$\tau$ & $e_{\tau}$ & $h_{\tau}$ \\
\hline load_M4 & $x_{M 4}=I$ & $x_{M 4}:=W$ \\
\hline tr_G I $_{-} A_{-}$pick & $x_{M 4}=W$ & $x_{M 4}:=I$ \\
\hline
\end{tabular}

As in FRP and SC, the four machine models possess similar (discrete) dynamics.

- Robot-1 (R1):

$\mathcal{V}_{R 1}=\left\{x_{R 1}, q_{B 1}, x_{A C}, \eta, t\right\}$

$\mathcal{T}_{R 1}=\left\{t r_{-} F_{-} B_{-}\right.$pick, $t r_{-} F_{-} B_{-} d r o p, t r_{-} F_{-} A_{-} p i c k, t r_{-} F_{-} A_{-} d r o p$,

Check_c1, $1 ? q_{B 1}$, initial, tick

$\Theta_{R 1}=\left(\eta=\right.$ initial $\left.\wedge x_{R 1}=I\right)$

\begin{tabular}{|c|l|c|}
\hline$\tau$ & $e_{\tau}$ & $h_{\tau}$ \\
\hline Check-c1 & $x_{R 1}=A \wedge q_{B 1}=F$ & $x_{R 1}:=I$ \\
\hline$c 1 q_{B 1}$ & $x_{R 1}=I$ & $\left(q_{B 1}:=x_{B 1}\right) \wedge\left(x_{R 1}:=A\right)$ \\
\hline$t r_{-} F_{-} B_{-}$pick & $\left(x_{R 1}=A\right) \wedge \sim\left(q_{B 1}=F\right)$ & $x_{R 1}:=W 1$ \\
\hline$t r_{-} F_{-} B_{-}$drop & $x_{R 1}=W 1$ & $x_{R 1}:=I$ \\
\hline$t r_{-} F_{-} A_{-}$pick & $\left(x_{R 1}=A \vee x_{R 1}=I\right)$ & $x_{R 1}:=W 2$ \\
\hline$t r_{-} F_{-} A_{-}$drop & $x_{R 1}=W\left(x_{A C}=E\right)$ & $x_{R 1}:=I$ \\
\hline
\end{tabular}

- Robot-2 (R2):

$\mathcal{V}_{R 2}=\left\{x_{R 2}, q_{B 2}, \eta, t\right\}$

$\mathcal{T}_{R 2}=\left\{t r_{-} G_{-} B_{-}\right.$pick, tr- $G_{-} B_{-} d r o p, t r_{-} G_{-} A_{-}$pick, tr $G_{-} G_{-} A_{-} d r o p$,

st_assm, fin_assm, Check_c2,c2?q $q_{B 2}$, initial, tick $\}$

$\Theta_{R 2}=\left(\eta=\right.$ initial $\left.\wedge x_{R 2}=I\right)$

\begin{tabular}{|c|c|c|}
\hline$\tau$ & $e_{\tau}$ & $h_{\tau}$ \\
\hline Check-c2 & $x_{R 2}=A \wedge q_{B 2}=F$ & $x_{R 2}:=I$ \\
\hline$c 2 ?_{B 2}$ & $x_{R 2}=I$ & $\left(q_{B 2}:=x_{B 2} \wedge\left(x_{R 2}:=A\right)\right.$ \\
\hline$t r_{-} G_{-} B_{-}$pick & $\left(x_{R 2}=A\right) \wedge \sim\left(q_{B 2}=F\right)$ & $x_{R 2}:=W 1$ \\
\hline$t r_{-} G_{-} B_{-}$drop & $x_{R 2}=W 1$ & $x_{R 2}:=I$ \\
\hline$t r_{-} G_{-} A_{-}$pick & $x_{R 2}=A \vee x_{R 2}=I$ & $x_{R 2}:=W 2$ \\
\hline$t r_{-} G_{-} A_{-}$drop & $x_{R 2}=W 2$ & $x_{R 2}:=I$ \\
\hline st_assm & $x_{R 2}=A \vee x_{R 2}=I$ & $x_{R 2}:=W 3$ \\
\hline fin_assm & $x_{R 2}=W 3$ & $x_{R 2}:=I$ \\
\hline
\end{tabular}


Here note that, in $R 1$ and in $R 2$, the local variables $q_{B 1}$ and $q_{B 2}$ get assigned to values of corresponding buffer states via communicating events. On the other hand, the activity variable of the assembly cell $x_{A C}$ is modelled as a shared variable between $A C$ and $R 2$. Further, note that, in both robot models, extra state and transitions have become necessary because of explicit communication through events.

- Assembly cell (AC):

$\mathcal{V}_{A C}=\left\{x_{A C}, \eta, t\right\}$

$\mathcal{T}_{A C}=\left\{t r_{-} F_{-} A_{-} d r o p, t r_{-} G_{-} A_{-} d r o p, s t_{-}\right.$assm, fin_assm, initial, tick $\}$

$\Theta_{A C}=\left(\eta=\right.$ initial $\left.\wedge x_{A C}=E\right)$

\begin{tabular}{|c|c|c|}
\hline$\tau$ & $e_{\tau}$ & $h_{\tau}$ \\
\hline$t r_{-} F_{-} A_{-}$drop & $x_{A C}=E$ & $x_{A C}:=F$ \\
\hline$t r_{-} G_{-} A_{-}$drop & $x_{A C}=F$ & $x_{R 2}:=F G$ \\
\hline st_assm & $x_{A C}=F G$ & $x_{A C}:=B$ \\
\hline fin_assm & $x_{A C}=B$ & $x_{A C}:=E$ \\
\hline
\end{tabular}

Finally, the manufacturing cell model is given by M.Cell $=M_{-} 1\left\|M_{-} 2\right\| M_{-} 3\left\|M_{-} 4\right\| B_{-} 1\left\|B_{-} 2\right\| R_{-} 1\left\|R_{-} 2\right\| R M \| A C$.

This appears similar to the case of FRP. However the role played by GCOs in the FRP model is automatically fulfilled by parallel composition of TTMs, as the transitions sharing identical names get composed into a single unique transition, bearing a combined enabling condition, under concurrent operation. Such synchronization is however constant unlike in FRP where it is dependent on the local alphabet.

\subsection{Remarks}

From the exercise of modelling the automated manufacturing system using different frameworks, the following points that emerge clearly are mentioned below.

(i) Synchronization is a major issue in modelling and is tackled differently in different models.

In PN model synchronisation is modelled by enabling a transition with multiple input places. So any system having some internal synchronisation has to be modelled as a whole, as if it is hardwired.

In FRP model the PCO models concurrency. The variable alphabet of processes is both a power as well as cause of complexity in this model. Note how the LCO $\left[+\left\{t r_{-} F_{-} B_{-} p i c k\right\}\right]$ is used in the definition of buffer 1 to prevent the robot 1 from picking up object from machine 1 , when the buffer is full. Also GCO is used over each component in the final definition of the manufacturing cell, to force global synchronization among different events. As mentioned before, we keep the basic component models unchanged, i.e., they maintain their independent operation and impose additional synchronization through LCO and GCO whenever required. This is preferable from the point of view of maintainability and reusability of models than including the alphabet into the local alphabet of core models everywhere. 
In SC model, like FRP, mere identity of names does not ensure synchronization. However instead of GCO and SCO, state based conditions have been used to ensure synchronisation as well as blocking.

In TTM, however, identically named transitions are destined to occur synchronously. This is because such transitions are fused into a new synchronized transition in the concurrent combination. As a result, unlike SC, conditions defined on most of the component transitions are local.

(ii) Communication between processes is explicitly modelled only in TTM, using both communication via channels $\left(c_{1}\right.$ and $\left.c_{2}\right)$ as well as shared variables $\left(x_{A C}\right.$ in $\left.\mathcal{V}_{R 1}\right)$. $\mathrm{PN}, \mathrm{SC}$ and FRP have no such features.

(iii) Modelling of the buffer shows interesting differences between the models. In PN the presence of 'tokens' takes care of the numerical value of buffer capacity. In TTM there is provision of explicit data variables. In SC and FRP, however modelling of buffer is cumbersome. In SC the number of basic states (under the $B i P F, i=1,2$ superstates) and correspondingly in FRP the number of equations describing the two buffers increases with increase of buffer capacity.

(iv) Hierarchy is explicitly treated in SC framework. In TTM and FRP it is achieved via modular design of components and PCO. In the final PN model the presence of hierarchy is not apparent. Zhou et al (1992) have shown how the final model is arrived at via stepwise refinement from simpler models of PN. This stepwise refinement strategy is however not inherent in the PN modelling tools and has to be designed by the modeller to suit his purpose.

(v) Other than TTM and SC, none of the above models can capture real-time features. But we have ignored timing features by taking lower and upper time bounds of all the transitions as 0 and $\infty$ respectively. It may be mentioned that performance questions related to timing are difficult to answer analytically and are usually dealt with by exhaustive automated simulations.

(vi) An FSM model for the example becomes unmanageable because of the large size of the state space as can easily be understood from the following argument.

From the statecharts model in figure 11, we note that for buffers of capacity four only, the product automata with no synchronization will have $2 \times 2 \times 2 \times 2 \times 5 \times 5 \times$ $3 \times 4 \times 2 \times 2 \times 4=38400$ states! However due to synchronization present a fraction of this only will exist in the concurrent behaviour. It is difficult to estimate the exact number of states manually, but even if only about ten percent of the states remain in the final model, it would be a staggering 4000 . Naturally, the FSM example has not been provided here.

\section{Comparison and conclusions}

\subsection{Comparison}

We have come across various types of modelling frameworks, each of which has certain strengths as well as weaknesses. The more powerful a modelling framework is, the more 
difficult it is to answer questions of reachability, boundedness, etc. in that setup. In fact, many such aspects of the more powerful models are undecidable. In order to get an overview of the various modelling frameworks, we present a table at the end of this section comparing them on the basis of the answers to the following questions:

Q1. Is the reachability problem decidable?

Q2. Is the liveness/deadlock problem decidable?

Q3. What is the language complexity?

Q4. Can it handle nondeterministic behaviour?

Q5. Does it support hierarchical structure?

Q6. Is the problem of control well-solved?

Q7. Is the problem of observation well-solved?

Q8. Is the problem of stabilization well-solved?

Q9. Can real-time features be handled?

Q10. Do there exist good analytical tools for analysing the model?

The answers in most cases are Y (Yes), QY (Qualified Yes), N (No) and ? (Unknown). Question 3 has however the following possible answers: R (Regular), CF (Context free), CS (Context sensitive) and RE (Recursively enumerable). The frameworks, which are compared, are the FSM, TTM. PN, Statecharts and FRP. Since we are often concerned with finite state versions of the first four systems, most of the questions related to them are decidable. Things are exactly the opposite for the last three. Activities on the control, and stabilization are till now restricted only to the first four, although not yet well-solved in all of them. Hierarchy is explicitly supported inherently only in the Statecharts model, while real time features are inherent only in the TTM. Such features have however been imposed on some of the other frameworks as well. Finally, graph and set-theoretic tools are available for the first four frameworks, in addition to temporal logic for TTM, while for the last three only logic and proof rules can be applied.

\begin{tabular}{|l|l|l|l|l|l|l|l|l|l|l|}
\hline Model & Q1 & Q2 & Q3 & Q4 & Q5 & Q6 & Q7 & Q8 & Q9 & Q10 \\
\hline FSM & Y & Y & R & Y & N & Y & Y & Y & QY & Y \\
\hline TTM & QY & QY & RE & Y & QY & QY & N & N & Y & Y \\
\hline PN & Y & Y & RE & Y & N & Y & QY & QY & Y & Y \\
\hline SC & Y & QY & $?$ & Y & Y & QY & QY & QY & QY & Y \\
\hline FRP & N & N & RE & N & QY & N & N & N & QY & QY \\
\hline
\end{tabular}

\subsection{Conclusion and future scope}

It is too early to say anything definitive about the future of DES research. While efforts are on to equip existing frameworks with new modelling features, a single model is unlikely to be appropriate for all situations. Experience of modelling large real-life systems is still lacking. Further developments in this field should be driven by practical problems. 
Some of the major issues that need to be addressed are classified as follows:

- Implementation: (i) How to handle the myriad of information about a complex system and make the necessary level of abstraction. (ii) How to move up and down in the abstraction hierarchy and automatically generate low level details from high level specification and other data. (iii) How to represent the derived model in the most appropriate form in the computer, so that it facilitates the use of the encoded information in some optimal way. (iv) How to simulate model behaviour.

- Specification and verification: (i) How to check whether the behaviour of the model conforms to certain hypotheses. (ii) How to specify the hypotheses themselves so that it becomes easy to verify them.

- Control: If the system does not satisfy the requirement, how to change its behaviour so that it does.

- Observation: (i) How to estimate system behaviour in the face of partial observation. (ii) How to control such a situation. (iii) What is the role of nondeterminism?

These questions, and many others, we feel, should keep the field active for at least some years to come.

\section{References}

Apt K R, Francez N, de Roever W P 1980 A proof system for communicating sequential processes. ACM Trans. Program. Languages Syst. 2: 359-385

Ausfelder C, Castelain E, Gentina J C 1994 A method for hierarchical modelling of the command of flexible manufacturing systems. IEEE Trans. Syst. Man Cybern. 24: 564-573

Bose S. Mukhopadhyay S 1995 An extended finitely recursive process model for discrete event systems. IEEE Trans. Syst. Man Cybern. 25: 1616-1627

Bose S. Patra A, Mukhopadhyay S 1994 On observability with delay: Antitheses and syntheses. IEEE Trans. Autom. Control 39: 803-806

Bose S, Mukhopadhyay S, Patra A 1995a Finiteness tharacterisation of finitely recursive processes. IEEE Trans. Autom. Control (communicated)

Bose S, Mukhopadhyay S, Patra A 1995b A nondeterministic extension of the finitely recursive process model. Discrete Event Dynamic Syst: Theor. Appl. (communicated)

Bose S, Patra A, Mukhopadhyay S 1995c Boundedness characterisation of finitely recursive processes. IEEE Trans. Autom. Control (communicated)

Brandt R D, Garg V, Kumar R, Lin F, Marcus S I, Wonham W M 1990 Formulas for calculating supremal controllable and normal sublanguages. Syst. Control Lett. 15: 111-117

Brave Y, Heymann M 1990 Stabilization of discrete event processes. Int. I. Control 51: 1101-1117

Brave Y, Heymann M 1993 Control of discrete event systems modeled as hierarchical state machines. IEEE Trans. Autom. Control 38: 1803-1819

Brookes S D, Roscoe A W 1991 Deadlock analysis in networks of communicating processes. Distributed Computing 4: 209-230

Chandy K M, Mishra J 1979 Deadlock absence proofs for networks of communicating processes. Inf. Process. Lett. 9: 185-189

Chen E, Lafortune S 1991 On nonconflicting languages that arise in supervisory control of discrete event systems. Syst. Control Lett. 17: 105-113 
Chung S L4, Lafortune S, Lin F 1992 Limited lookahead policies in supervisory control of discrete event systems. IEEE Trans. Autom. Control 37: 1921-1935

Cieslak R A, Varaiya P 1990 Undecidability results for deterministic communicating sequential processes. IEEE Trans. Autom. Control 35: 1032-1039

Cieslak R A. Desclaux C, Fawaz A S, Varaiya P 1988 Supervisory control of discrete event processes with partial observations. IEEE Trans. Autom. Control 33: 249-260

Clarke E M. Emerson E A, Sistla A P 1986 Automatic verification of finite state concurrent systems using temporal logic specifications. ACM Trans. Program. Languages Syst. $8(2)$ :

Dathi N 1989 Deadlock and deadlock-freedom. Ph D thesis, Oxford University Computing Laboratory

David R, Alla H 1994 Petri Nets for modelling of dynamic systems - A survey. Automatica 30: 175-202

Drusinsky D 1991 A state assignment procedure for single-block implementation of Statecharts. IEEE Trans. Comput. Aided Design 10: 1569-1576

Drusinsky D, Harel D 1989 Using Statecharts for hardware description and synthesis. IEEE Trans. Comput. Aided Design 8: 798-807

Ferrarini L 1992 An incremental approach to logic controller design with Petri Nets. IEEE Trans. Syst. Man Cybern. 22: 461-473

Ferrarini L, Narduzzi M, Tassan-Solet M 1994 A new approach to modular liveness conceived for large logic controller design. IEEE Trans. Robot. Autom. 10: 169-184

Floyd R W 1967 Assigning meaning to programs. In Proceedings of Symposia in Applied Mathematics (Providence, Rhode Island: Am. Math. Soc.) vol. 19, pp 19-32

Harel D, Pnueli A. Schmidt J P, Sherman R 1987 On the formal semantics of statecharts. In Proc. of 2nd IEEE Symp. on Logic in Computer Science pp 54-64

Harel D, Lachover H, Naamad A. Pnueli A. Politi M, Sherman R, Shtull-Trauring A, Trakhtenbrot M 1990 STATEMATE: A working environment for the development of complex reactive systems. IEEE Trans. Software Eng. 16: 403-413

Hennessy M 1988 Algebraic theor of processes (Cambridge, MA: MIT Press)

Heymann M, Lin F 1993 Online control of partially observed discrete event systems. Proc. European Control Conference pp 2169-2174

Ho Y C 1989 Scanning the issue: Editorial. Proc. IEEE (Special issue on discrete event dynamic systems) 77: 3-6

Hoare C A R 1969 An axiomatic basis for computer programming. Commun. ACM 12: 576

Hoare C A R 1985 Communicating sequential processes (New Delhi: Prentice Hall India)

Holloway L E, Krogh B H 1990 Synthesis of feedback control logic for a class of controlled Petri Nets. IEEE Trans. Autom. Control 35: 514-523

Holt R C 1972 Some deadlock properties of computer systems. ACM Comput. Surv. 4: 179-196 Hopcroft J E. Ullman J D 1979 Introduction to automata theory: Languages and computation (Reading, MA: Addison-Wesley)

iLOGIX Corporation, The semantics of statecharts

iLOGIX Corporation, The languages of STATEMATE

Inan $\mathrm{K}$, Varaiya $\mathrm{P} 1988$ Finitely recursive process models for discrete event systems. IEEE Trans. Autom. Control 33: 626-639

Knight J F, Passino K M 1990 Decidability for a temporal logic used in discrete-event systems analysis. Int. J. Control 52: 1489-1506

Kozák P, Wonham W M 1995 Fully decentralized solutions of supervisory control problems. IEEE Trans. Autom. Control 40: 2094-2097 
Kumar R, Garg V, Marcus S I 1991 On controllability and normality of discrete-event dynamic systems. Syst. Control Lett. 17: 157-168

Kumar R, Garg V, Marcus S I 1993a On language stability and stabilizability of discrete event dynamic systems. SIAM J. Control Optimization 31: 1294-1320

Kumar R, Garg V, Marcus S I 1993b Predicates and predicate transformers for supervisory control of discrete event dynamic systems. IEEE Trans. Autom. Control 38: 232-247

Kumar R. Garg V, Marcus S I 1995 Finite buffer realization of input-output discrete-event systems. IEEE Trans. Autom. Control 40: 1042-1053

Lafortune S, Chen E 1990 The infimal closed controllable superlanguage and its application in supervisory control. IEEE Trans. Autom. Control 35: 398-405

Lawford M, Wonham W M 1995 Equivalence preserving transformations for timed transition models. IEEE Trans. Autom. Control 40: 1167-1179

Lin F 1991 Control of large scale discrete event systems: Task allocation and coordination. Syst. Control Lett. 17: 169-175

Lin F, Wonham W M 1988a On observability of discrete event systems. Inf. Sci. 44: 173-198

Lin F, Wonham W M 1988 b Decentralized supervisory control of discrete event systems. Inf. Sci. 44: $199-224$

Lin F. Wonham W M 1990 Decentralized control and coordination of discrete event systems with partial observation. IEEE Trans. Autom. Controi 35: 1330-1337

Lin F, Wonham W M 1991 Verification of nonblocking in decentralized supervision. Control Theor. Adv. Technol. 7: 19-29

Manna Z, Pnueli A 1982 Verification of concurrent programs: The temporal framework. In The correctness problem in computer science, International Lecture Series in Computer Science (eds) R S Boyer, J S Moore (London: Academic Press) pp 215-273

Maxemchuck N F, Sabnani K 1987 Probabilistic verification of communication protocols. In Protocol specification, testing and verification (eds) $\mathrm{H}$ Rudin, $\mathrm{CH}$ West (Amsterdam: NorthHolland) vol. 7, pp 307-320

Murata T 1989 Petri Nets: Properties, analysis and applications. Proc. IEEE 77: 541-580

Ostroff J S 1990 Deciding properties of timed transition models. IEEE Trans. Parallel Distributed Syst. 1: 170-183

Ostroff J S, Wonham W M 1990 A framework for real-time discrete event control. IEEE Trans. Autom. Control 35: 386-397

Özveren C M, Willsky A S 1990 Observability of discrete event dynamic systems. IEEE Trans. Autom. Control 35: 797-806

Özveren C M, Willsky A S 1991 Output stabilizability of discrete event dynamic systems. IEEE Trans. Autom. Control 36: 925-935

Özveren C M, Willsky A S 1992a Invertibility of discrete event dynamic systems. Math. Controls, Signals Syst. 5: 365-390

Özveren C M, Willsky A S $1992 b$ Tracking and restrictability in discrete event dynamic systems. SIAM J. Control Optimization 30: 1432-1446

Özveren C M, Willsky A S 1992c Aggregation and multilevel control in discrete event dynamic systems. Automatica 28: 565-577

Özveren C M, Willsky A S, Antsaklis P J 1991 Stability and stabilizability of discrete event dynamic systems. J. Assoc. Comput. Mach. 38: 730-752

Passino K M 1989 Analysis and synthesis of discrete event regulator systems. $\mathrm{Ph} D$ thesis, Department of Electrical Engineering, University of Notre Dame. Notre Dame, IN

Peterson J L 1981 Petri net theory and modeling of systems (Englewood Cliffs, NJ: Prentice Hall) 
Petri C A 1962 Kommunikation mit Automaten. Ph D thesis, Institut für Instrumentelle Mathematik, Bonn

Ramadge P J G, Wonham W M 1987a Supervisory control of a class of discrete event processes. SIAM J. Control Optimization 25: 206-230

Ramadge P J G, Wonham W M 1987b Modular feedback logic for discrete event systems. SIAM J. Control Optimization 25: 1202-1218

Ramadge P J G, Wonham W M 1989 The control of discrete event systems, Proc. IEEE (Special Issue on Discrete Event Dynamic Systems) 77: 81-98

Rudie K, Willems J C 1995 The computational complexity of decentralized discrete event control problems. IEEE Trans. Autom. Control 40: 1313-1319

Rudie K, Wonham W M 1990 The infimal prefix-closed and observable superlanguage of given language. Syst. Control Lett. 17: 361-371

Rudie K. Wonham W M 1992 Think locally, act globally: Decentralized supervisory control. IEEE Trans. Autom. Control 37: 1692-1708

Sampath M, Sengupta R, Lafortune S, Sinnamohideen K. Teneketzis D 1995 Diagnosability of discrete-event systems. IEEE Trans. Autom. Control 40: 1555-1575

Takai S, Ushio T, Kodama S 1995 Static state feedback control of discrete-event systems under partial observation. IEEE Trans. Autom. Control 40: 1950-1954

Thistle J G, Wonham W M 1986 Control problems in a temporal logic framework. Int. J. Control 44: 943-976

Tsitsiklis J N 1989 On the control of discrete event dynamic systems. Math. Controls, Signals Syst. 2: 95-107

Ushio T 1990 Feedback logic for discrete event systems with arbitrary control patterns. Int. J. Control 52: 159-174

Willner Y, Heymann M 1991 Supervisory control of concurrent discrete event systems. Int. $J$. Control 54: 1145-1169

Wonham W M, Ramadge P J G 1987 On the supremal controllable sublanguage of a given language. SIAM J. Control Optimization 25: 637-659

Wonham W M, Ramadge P J G 1988 Modular supervisory control of discrete event systems. Math. Controls, Signals Syst. 1: 13-30

Yang X, Lemmon M D, Antsaklis P J 1995 On the supremal controllable sublanguage in the discrete-event model of nondeterministic hybrid control systems IEEE Trans. Autom. Control 40: 2098-2103

Zhong H, Wonham W M 1990 On the consistency of hierarchical supervision in discrete event systems. IEEE Trans. Autom. Control 35: 1125-1134

Zhou M C, DiCesare F. Desrochers A A 1992 A hybrid methodology for synthesis of Petrinet models for manufacturing systems. IEEE Trans. Robot. Autom. 8: 350-361

Zhou M C, McDermott K. Patel P A 1993 Petrinet analysis and synthesis of a flexible manufacturing system cell. IEEE Trans. Svst., Man Cybern. 23: 523-531 